\title{
ABSTRACT INTERPOLATION PROBLEM IN NEVANLINNA CLASSES
}

\author{
VLADIMIR DERKACH
}

\begin{abstract}
The abstract interpolation problem (AIP) in the Schur class was posed V. Katznelson, A. Kheifets and P. Yuditskii in 1987 as an extension of the V.P. Potapov's approach to interpolation problems. In the present paper an analog of the AIP for Nevanlinna classes is considered. The description of solutions of the AIP is reduced to the description of L-resolvents of some model symmetric operator associated with the AIP. The latter description is obtained by using the M.G. Krein's theory of L-resolvent matrices. Both regular and singular cases of the AIP are treated. The results are illustrated by the following examples: bitangential interpolation problem, full and truncated moment problems. It is shown that each of these problems can be included into the general scheme of the AIP.
\end{abstract}

\section{INTRODUCTION}

The abstract interpolation problem (AIP) was posed by V. Katznelson, A. Kheifets and P. Yuditskii in [25] as an extension of the V.P. Potapov's approach to interpolation problems [27]. In a sense the problem consists in contractive "embedding" of some partial isometry $V$ acting in a structured Hilbert space $\mathcal{H} \oplus \mathcal{L}$ into a model unitary operator acting in a space $\mathcal{H}^{w} \oplus \mathcal{L}$, where $\mathcal{H}^{w}$ is the de Branges-Rovnyak space corresponding to an operator valued function (ovf) $w$ from the Schur class $S(\mathcal{L})$. A description of the set of all ovf $w \in S(\mathcal{L})$ for which such an "embedding" is possible were reduced in [25] to the description of all scattering matrices of unitary extensions of a given partial isometry $V([7])$. In a number of papers it was shown that many problems of analysis, such that the bitangential interpolation problem [26], moment problem [29], lifting problem [34], and others can be included into the general scheme of AIP.

In the present paper we consider a parallel version of AIP for Nevanlinna class. The class $N[\mathcal{L}]$ consists of all ovf on $\mathbb{C}_{+} \cup \mathbb{C}_{-}$with values in the set $[\mathcal{L}]$ of bounded linear operators in $\mathcal{L}$ such that $m(\bar{\lambda})=m(\lambda)^{*}$ and the kernel

$$
\mathrm{N}_{\omega}^{m}(\lambda)=\frac{m(\lambda)-m(\omega)^{*}}{\lambda-\bar{\omega}}
$$

is nonnegative on $\mathbb{C}_{+}$. Then the kernel $\mathbb{N}_{\omega}^{m}(\lambda)$ is also nonnegative on $\mathbb{C}_{+} \cup \mathbb{C}_{-}$.

Date: October 19, 2018.

1991 Mathematics Subject Classification. Primary 47A57; Secondary 30E05, 47A06, 47B25, 47B32.

Key words and phrases. Symmetric relation, selfadjoint extension, reproducing kernel, abstract interpolation problem, boundary triplet, resolvent matrix, moment problem.

This research has been done partially while the author was visiting the Department of Mathematics of Weizmann Institute of Science as a Weston Visiting Scholar . 
In introduction we restrict ourselves to the case when $\operatorname{dim} \mathcal{L}<\infty$ and identify $\mathcal{L}$ with the space $\mathbb{C}^{d}$ where the standard basis is chosen. Then every ovf $m \in N^{d \times d}:=$ $N\left[\mathbb{C}^{d}\right]$ can be considered as a $d \times d$ matrix valued function $(\operatorname{mvf})$. Let $\mathcal{H}(m)$ be the reproducing kernel Hilbert space (see [13], [4]) which is characterized by the properties:

(1) $\mathrm{N}_{\omega}^{m}(\lambda) u \in \mathcal{H}(m)$ for all $\omega \in \mathbb{C} \backslash \mathbb{R}$ and $u \in \mathbb{C}^{d}$;

(2) for every $f \in \mathcal{H}(m)$ the following identity holds

$$
\left\langle f(\cdot), \mathrm{N}_{\omega}^{m}(\lambda) u\right\rangle_{\mathcal{H}(m)}=u^{*} f(\omega), \quad \omega \in \mathbb{C} \backslash \mathbb{R}, u \in \mathbb{C}^{d} .
$$

The AIP in the class $N^{d \times d}$ can be formulated as follows.

Let $\mathcal{X}$ be a complex linear space, let $B_{1}, B_{2}$ be linear operators in $\mathcal{X}$, let $C_{1}, C_{2}$ be linear operators from $\mathcal{X}$ to $\mathbb{C}^{d}$, and let $K$ be a nonegative sesquilinear form on $\mathcal{X}$ which satisfies the following identity

(A1) $K\left(B_{2} h, B_{1} g\right)-K\left(B_{1} h, B_{2} g\right)=\left(C_{1} h, C_{2} g\right)_{\mathbb{C}^{d}}-\left(C_{2} h, C_{1} g\right)_{\mathbb{C}^{d}}$

for every $h, g \in \mathcal{X}$. Consider the following

Problem $A I P\left(B_{1}, B_{2}, C_{1}, C_{2}, K\right)$. Let the data set $\left(B_{1}, B_{2}, C_{1}, C_{2}, K\right)$ satisfies the assumption $(A 1)$. Find a mvf $m \in N^{d \times d}$ such that for some linear mapping $F$ : $X \rightarrow \mathcal{H}(m)$ the following conditions hold:

$(\mathrm{C} 1)\left(F B_{2} h\right)(\lambda)-\lambda\left(F B_{1} h\right)(\lambda)=\left[\begin{array}{ll}I & -m(\lambda)\end{array}\right]\left[\begin{array}{l}C_{1} h \\ C_{2} h\end{array}\right]$;

(C2) $\|F h\|_{\mathcal{H}(m)}^{2} \leq K(h, h)$

for all $h \in \mathcal{X}$.

Clearly ker $K=\{h \in \mathcal{X}: K(h, h)=0\}$ is a linear subspace of $\mathcal{X}$. Let $\mathcal{H}$ be the completion of the factor-space $\widehat{\mathcal{X}}=\mathcal{X} /$ ker $K$ endowed with the scalar product

$$
(\widehat{h}, \widehat{g})_{\mathcal{H}}=K(h, g), \quad \widehat{h}=h+\operatorname{ker} K, \widehat{g}=g+\operatorname{ker} K, h, g \in \mathcal{X} .
$$

It follows from (A1) that the linear relation

$$
\widehat{A}=\left\{\left\{\left[\widehat{B_{1} h},\left[\begin{array}{l}
\widehat{B_{2} h} \\
C_{1} h
\end{array}\right]\right\}: h \in \mathcal{X}\right\}\right.
$$

is symmetric in $\mathcal{H} \oplus \mathbb{C}^{d}$. The main result of the paper is the following description of all the AIP solutions $m$.

Theorem 1. Let the data set $\left(B_{1}, B_{2}, C_{1}, C_{2}, K\right)$ satisfies the assumption $(A 1)$ and let $\operatorname{ran} C_{2}=\mathcal{L}=\mathbb{C}^{d}$. Then the Problem $A I P\left(B_{1}, B_{2}, C_{1}, C_{2}, K\right)$ is solvable and the set of its solutions is parametrized by the formula

$$
\left[\begin{array}{c}
m(\lambda) \\
I_{d}
\end{array}\right]=\left[\begin{array}{cc}
I_{d} & 0 \\
\lambda & I_{d}
\end{array}\right]\left[\begin{array}{c}
P_{\mathcal{L}}(\widetilde{A}-\lambda)^{-1} I_{\mathcal{L}} \\
I_{\mathcal{L}}
\end{array}\right]\left(I_{d}+\lambda P_{\mathcal{L}}(\widetilde{A}-\lambda)^{-1} I_{\mathcal{L}}\right)^{-1}
$$

where $\widetilde{A}$ ranges over the set of all selfadjoint extensions of $\widehat{A}$ with the exit in a Hilbert space $\widetilde{\mathcal{H}} \oplus \mathcal{L} \supset \mathcal{H} \oplus \mathcal{L}$. The corresponding linear mapping $F: X \rightarrow \mathcal{H}(\varphi, \psi)$ is given by

$$
(F h)(\lambda)=P_{\mathcal{L}}(\widetilde{A}-\lambda)^{-1} \widehat{h}, \quad h \in X .
$$

Due to Theorem 1 the description of all solutions $m$ of the $A I P\left(B_{1}, B_{2}, C_{1}, C_{2}, K\right)$ is reduced to the problem of description of all $\mathcal{L}$-resolvents of the linear relation $\widehat{A}$. The latter description has been obtained by M.G. Krĕ̌n in [31] (see also [33]). 
In order to apply this theory to the linear relation $\widehat{A}$ we will impose additional assumptions on the data set $\left(B_{1}, B_{2}, C_{1}, C_{2}, K\right)$.

(A2) $\operatorname{dim} \operatorname{ker} K<\infty$ and $\mathcal{X}$ admits the representation

$$
\mathcal{X}=\mathcal{X}_{0}+\operatorname{ker} K
$$

such that $B_{j} \mathcal{X}_{0} \subseteq \mathcal{X}_{0}(j=1,2)$.

(A3) $B_{2}=I_{\mathcal{X}}$ and the operators $\left.B_{1}\right|_{\mathcal{X}_{0}}: \mathcal{X}_{0} \subset \mathcal{H} \rightarrow \mathcal{H},\left.C_{1}\right|_{\mathcal{X}_{0}},\left.C_{2}\right|_{\mathcal{X}_{0}}: \mathcal{X}_{0} \subset \mathcal{H} \rightarrow \mathcal{L}$ are bounded.

The continuations of the operators $\left.B_{1}\right|_{\mathcal{X}_{0}},\left.C_{1}\right|_{\mathcal{X}_{0}},\left.C_{2}\right|_{\mathcal{X}_{0}}$ will be denoted by $\widetilde{B}_{1} \in[\mathcal{H}]$, $\widetilde{C}_{1}, \widetilde{C}_{2} \in[\mathcal{H}, \mathcal{L}]$.

Denote by $\widetilde{N}^{d \times d}$ the set of Nevanlinna pairs $\{p, q\}$ of $d \times d \operatorname{mvfs} p(\cdot), q(\cdot)$ holomorphic on $\mathbb{C} \backslash \mathbb{R}$ such that:

(i) the kernel $\mathrm{N}_{\omega}^{p, q}(\lambda)=\frac{q(\bar{\lambda})^{*} p(\bar{\omega})-p(\bar{\lambda})^{*} q(\bar{\omega})}{\lambda-\bar{\omega}}$ is nonnegative on $\mathbb{C}_{+}$;

(ii) $q(\bar{\lambda})^{*} p(\lambda)-p(\bar{\lambda})^{*} q(\lambda)=0, \lambda \in \mathbb{C} \backslash \mathbb{R}$;

(iii) $0 \in \rho(p(\lambda)-\lambda q(\lambda)), \lambda \in \mathbb{C}_{ \pm}$.

In the regular case $(\operatorname{ker} K=\{0\})$ the set of $\mathcal{L}$-resolvents $\left.P_{\mathcal{L}}(\widetilde{A}-\lambda)^{-1}\right|_{\mathcal{L}}$ of $\widehat{A}$ can be described by the formula (see [30], 333])

$$
P_{\mathcal{L}}(\widetilde{A}-\lambda)^{-1} I_{\mathcal{L}}=\left(\widehat{w}_{11}(\lambda) q(\lambda)+\widehat{w}_{12}(\lambda) p(\lambda)\right)\left(\widehat{w}_{21}(\lambda) q(\lambda)+\widehat{w}_{22}(\lambda) p(\lambda)\right)^{-1},
$$

where $\{p, q\} \in \widetilde{N}^{d \times d}$ and $\widehat{W}=\left[\widehat{w}_{i j}(\lambda)\right]_{i, j=1}^{2}$ is an $\mathcal{L}$-resolvent matrix of $\widehat{A}$ which can be calculated explicitly in terms of the data set (see (4.34)). Combining (1.4) and (1.7) one obtains the following

Theorem 2. Let the AIP data set $\left(B_{1}, B_{2}, C_{1}, C_{2}, K\right)$ satisfy $(A 1)$, (A3), ker $K=$ $\{0\}$ and $\operatorname{ran} C_{2}=\mathcal{L}=\mathbb{C}^{d}$ and let

$$
\Theta(\lambda)=\left[\begin{array}{ll}
\theta_{11}(\lambda) & \theta_{12}(\lambda) \\
\theta_{21}(\lambda) & \theta_{22}(\lambda)
\end{array}\right]=I_{\mathcal{L} \oplus \mathcal{L}}-\lambda\left[\begin{array}{l}
\widetilde{C}_{1} \\
\widetilde{C}_{2}
\end{array}\right]\left(I_{\mathcal{H}}-\lambda \widetilde{B}_{1}\right)^{-1}\left[\begin{array}{ll}
-\widetilde{C}_{2}^{+} & \widetilde{C}_{1}^{+}
\end{array}\right] .
$$

Then the formula

$$
m(\lambda)=\left(\theta_{11}(\lambda) q(\lambda)+\theta_{12}(\lambda) p(\lambda)\right)\left(\theta_{21}(\lambda) q(\lambda)+\theta_{22}(\lambda) p(\lambda)\right)^{-1},
$$

establishes the 1-1 correspondence between the set of all solutions $m$ of the AIP and the set of all equivalence classes of Nevanlinna pairs $\{p, q\} \in \widetilde{N}(\mathcal{L})$.

The operators $\widetilde{C}_{1}^{+}, \widetilde{C}_{2}^{+}$in (1.8) are adjoint operators to $\widetilde{C}_{1}, \widetilde{C}_{2}: \mathcal{H} \rightarrow \mathcal{L}$, that is

$$
\left(\widetilde{C}_{j}^{+} u, h\right)_{\mathcal{H}}=\left(u, \widetilde{C}_{j} h\right)_{\mathcal{L}} \quad(j=1,2, h \in \mathcal{H}, u \in \mathcal{L}) .
$$

Let the matrix $J \in \mathbb{C}^{2 d \times 2 d}$ be given by

$$
J=\left[\begin{array}{cc}
0 & -i I_{d} \\
i I_{d} & 0
\end{array}\right]
$$

The mvf $\Theta(\lambda)$ belongs to the Potapov class (see [37]), i.e. $\Theta(\lambda)$ has the following $J$-property

$$
\frac{J-W(\lambda) J W(\lambda)^{*}}{\lambda-\bar{\lambda}} \geq 0 \quad \text { for all } \lambda \in \mathbb{C} \backslash \mathbb{R}
$$


In the singular case the formula (1.7) gives a description of all $\mathcal{L}$-resolvents of the linear relation

$$
A_{0}=\left\{\left\{\left[\begin{array}{l}
B_{1} h \\
C_{1} h
\end{array}\right],\left[\begin{array}{l}
B_{2} h \\
C_{2} h
\end{array}\right]\right\}: h \in \mathcal{X}_{0}\right\} .
$$

To obtain a description of all $\mathcal{L}$-resolvents of $\widehat{A}\left(\supset A_{0}\right)$ one should consider in (1.7) only those Nevanlinna pairs $\{p, q\} \in \widetilde{N}(\mathcal{L})$ for which $\widetilde{A} \supset \widehat{A}\left(\supset A_{0}\right)$. We show that after the replacement in (1.9) of $\Theta(\lambda)$ by the $\Theta(\lambda) V$, where $V$ is an appropriate $J$-unitary matrix, the formula (1.9) gives a description of all the solutions of the AIP when $\{p, q\}$ ranges over the set of all Nevanlinna pairs of the form

$$
p(\lambda)=\left[\begin{array}{cc}
\widetilde{0}_{\nu} & 0 \\
0 & p_{1}(\lambda)
\end{array}\right], \quad q(\lambda)=\left[\begin{array}{cc}
\widetilde{I}_{\nu} & 0 \\
0 & q_{1}(\lambda)
\end{array}\right], \quad\left\{p_{1}, q_{1}\right\} \in \widetilde{N}^{d-\nu},
$$

where $\nu=\operatorname{dim} C$ ker $K$.

All these results are formulated in the paper in a more general situation, when the mvf $m$ is replaced by a Nevanlinna pair $\{\varphi, \psi\}$. Moreover, we do not suppose, in general, that $\operatorname{dim} \mathcal{L}<\infty$.

The paper is organized as follows. In Section 2 we recall the definition of the class $\widetilde{N}(\mathcal{L})$ of Nevanlinna pairs from [6, [18]. To each selfadjoint linear relation $\widetilde{A}$ and a scale spaces $\mathcal{L}$ we associate a Nevanlinna pair $\{\varphi, \psi\}$ by the formula

$$
\psi(\lambda):=\left.P_{\mathcal{L}}(\widetilde{A}-\lambda)^{-1}\right|_{\mathcal{L}}, \quad \varphi(\lambda):=I_{\mathcal{L}}+\left.\lambda P_{\mathcal{L}}(\widetilde{A}-\lambda)^{-1}\right|_{\mathcal{L}}, \lambda \in \rho(\widetilde{A}) .
$$

Conversely, given a Nevanlinna pair $\{\varphi, \psi\}$ normalized by the condition $p(\lambda)-$ $\lambda q(\lambda)=I_{d}$ we construct a functional model for a selfadjoint linear relation $\widetilde{A}(\varphi, \psi)$, such that the pair $\{\varphi, \psi\}$ is related to $\widetilde{A}(\varphi, \psi)$ via (1.11). In the case when the pair $\{\varphi, \psi\}$ is equivalent to a pair $\left\{I_{d}, m\right\}$ with $m \in N[\mathcal{L}]$, functional model $A(m)$ for this symmetric operator was given in [6] (see also [17]). Conditions when the model $A(\varphi, \psi)$ is reduced to $A(m)$ are discussed. In Sections 3 and 4 we formulate the AIP in the classes $N[\mathcal{L}]$ and $\widetilde{N}(\mathcal{L})$ and give a complete description of its solutions under some additional restrictions on the data set both in the regular and singular cases. The results of the paper are illustrated in Section 5 with an example of bitangential interpolation problems in the classes $N^{d \times d}$ and $\widetilde{N}^{d \times d}$, reduced there to the AIP with appropriately chosen data set. These problems have been studied earlier in [36], 6], [28], [20], [21], [12].

Mention, that the Arov and Grossman's description of scattering matrices of unitary extensions of an isometry $V$ in [7] used in the Schur type AIP is an analog of M.G. Kreľn's description (1.7) of $\mathcal{L}$-resolvents of a symmetric operator [30]. One of the goals of this paper is the formulation of the AIP, where the M.G. KreInn's formula (1.7) works directly. In particular, we use the example of the full moment problem to show that the reduction of this problem to the Nevanlinna type AIP is more natural and simpler than that in [29], where the reduction of the moment problem to the Schur type AIP was performed.

Another goal of the paper was to elaborate the operator approach to singular AIP. This approach is illustrated with an example of singular truncated moment problem, where we discuss the results of [11] and explain them from our point of view.

The paper is dedicated to the centennial of M.G. Krĕn. 


\section{FunCtional MOdel of a SELFAdjoint Linear RELAtion}

2.1. Nevanlinna pairs. Let $\mathcal{L}$ be a Hilbert space.

Definition 2.1. A pair $\{\Phi, \Psi\}$ of $[\mathcal{L}]$-valued functions $\Phi(\cdot), \Psi(\cdot)$ holomorphic on $\mathbb{C} \backslash \mathbb{R}$ is said to be a Nevanlinna pair if:

(i) the kernel

$$
\mathrm{N}_{\omega}^{\Phi, \Psi}(\lambda)=\frac{\Psi(\bar{\lambda})^{*} \Phi(\bar{\omega})-\Phi(\bar{\lambda})^{*} \Psi(\bar{\omega})}{\lambda-\bar{\omega}}, \quad \lambda, \omega \in \mathbb{C}_{+}
$$

is nonnegative on $\mathbb{C}_{+}$;

(ii) $\Psi(\bar{\lambda})^{*} \Phi(\lambda)-\Phi(\bar{\lambda})^{*} \Psi(\lambda)=0, \lambda \in \mathbb{C} \backslash \mathbb{R}$;

(iii) $0 \in \rho(\Phi(\lambda)-\lambda \Psi(\lambda)), \lambda \in \mathbb{C}_{ \pm}$.

Two Nevanlinna pairs $\{\Phi, \Psi\}$ and $\left\{\Phi_{1}, \Psi_{1}\right\}$ are said to be equivalent, if $\Phi_{1}(\lambda)=$ $\Phi(\lambda) \chi(\lambda)$ and $\Psi_{1}(\lambda)=\Psi(\lambda) \chi(\lambda)$ for some operator function $\chi(\cdot) \in[\mathcal{H}]$, which is holomorphic and invertible on $\mathbb{C}_{+} \cup \mathbb{C}_{-}$. The set of all equivalence classes of Nevanlinna pairs in $\mathcal{L}$ will be denoted by $\widetilde{N}(\mathcal{L})$. We will write, for short, $\{\Phi, \Psi\} \in$ $\widetilde{N}(\mathcal{L})$ for Nevanlinna pair $\{\Phi, \Psi\}$.

A Nevanlinna pair $\{\Phi, \Psi\}$ will be said to be normalized if $\Phi(\lambda)-\lambda \Psi(\lambda)=I_{\mathcal{H}}$. Clearly, every Nevanlinna pair $\{\Phi, \Psi\}$ is equivalent to the unique normalized Nevanlinna pair $\{\varphi, \psi\}$ given by

$$
\varphi(\lambda)=\Phi(\lambda)(\Phi(\lambda)-\lambda \Psi(\lambda))^{-1}, \quad \psi(\lambda)=\Psi(\lambda)(\Phi(\lambda)-\lambda \Psi(\lambda))^{-1} .
$$

The set $\widetilde{N}(\mathcal{L})$ can be identified with the set of Nevanlinna families (see [18])

$$
\tau(\lambda)=\{\{\Phi(\lambda) u, \Psi(\lambda) u\}: u \in \mathcal{L}\}, \quad\{\Phi, \Psi\} \in \widetilde{N}(\mathcal{L})
$$

Define the class $N(\mathcal{L})$ as the set of all Nevanlinna pairs $\{\Phi, \Psi\} \in \widetilde{N}(\mathcal{L})$ such that $\operatorname{ker} \Phi(\lambda)=\{0\}$ for some (and hence for all) $\lambda \in \mathbb{C} \backslash \mathbb{R}$. The set $N[\mathcal{L}]$ can be embedded in $N(\mathcal{L})$ via the mapping

$$
m \in N[\mathcal{L}] \mapsto\left\{I_{\mathcal{L}}, m\right\} \in N(\mathcal{L}) .
$$

\subsection{Nevanlinna pair corresponding to a selfadjoint linear relation and a} scale. Let $\mathfrak{H}, \mathcal{L}$ be Hilbert spaces, let $\widetilde{A}$ be a selfadjoint linear relation in $\mathfrak{H} \oplus \mathcal{L}$ and let $P_{\mathcal{L}}$ be the orthogonal projection onto the scale space $\mathcal{L}$. Define the operator valued functions

$$
\psi(\lambda):=\left.P_{\mathcal{L}}(\widetilde{A}-\lambda)^{-1}\right|_{\mathcal{L}}, \quad \varphi(\lambda):=I_{\mathcal{L}}+\left.\lambda P_{\mathcal{L}}(\widetilde{A}-\lambda)^{-1}\right|_{\mathcal{L}}, \lambda \in \rho(\widetilde{A}) .
$$

Proposition 2.2. The pair of operator valued functions $\{\varphi, \psi\}$, associated with a selfadjoint linear relation $\widetilde{A}$ and the scale $\mathcal{L}$ is a normalized Nevanlinna pair.

Proof. Consider the kernel

$$
\mathrm{N}_{\omega}^{\varphi \psi}(\lambda)=\frac{\psi(\bar{\lambda})^{*} \phi(\bar{\omega})-\phi(\bar{\lambda})^{*} \psi(\bar{\omega})}{\lambda-\bar{\omega}}, \quad \lambda, \omega \in \mathbb{C}_{+} \cup \mathbb{C}_{-} .
$$


It follows from (2.3)-(2.4) that

$$
\begin{aligned}
\mathrm{N}_{\omega}^{\varphi \psi}(\lambda) & =\frac{\psi(\lambda)-\psi(\omega)^{*}}{\lambda-\bar{\omega}}-\psi(\lambda) \psi(\omega)^{*} \\
& =\left.P_{\mathcal{L}} \frac{R_{\lambda}-R_{\bar{\omega}}}{\lambda-\bar{\omega}}\right|_{\mathcal{L}}-\left.P_{\mathcal{L}} R_{\lambda} P_{\mathcal{L}} R_{\bar{\omega}}\right|_{\mathcal{L}} \\
& =\left.P_{\mathcal{L}} R_{\lambda} P_{\mathcal{H}} R_{\bar{\omega}}\right|_{\mathcal{L}}
\end{aligned}
$$

and hence the kernel $\mathrm{N}_{\omega}^{\varphi \psi}(\lambda)$ is nonnegative.

The property (ii) is easily checked, $\varphi(\lambda)-\lambda \psi(\lambda)=I_{\mathcal{L}}$ and, hence, the pair $\{\phi, \psi\}$ is a normalized Nevanlinna pair.

Definition 2.3. The pair of operator valued functions determined by (2.3) will be called the Nevanlinna pair corresponding to the selfadjoint linear relation $\widetilde{A}$ and the scale $\mathcal{L}$.

Remark 2.4. Definition 2.3 is inspired by the notion of the Weyl family of a symmetric operator corresponding to a boundary relation, see [19]. Namely, the Nevanlinna pair $\{\varphi, \psi\}$ determined by (2.3) generates via (2.2) the Weyl family of the symmetric linear relation $S=\widetilde{A} \cap(\mathcal{H} \oplus \mathcal{H})$, corresponding to the boundary relation

$$
\Gamma=\left\{\left\{\left[\begin{array}{l}
f \\
f^{\prime}
\end{array}\right],\left[\begin{array}{c}
h^{\prime} \\
h
\end{array}\right]\right\}:\left\{\left[\begin{array}{l}
f \\
h
\end{array}\right],\left[\begin{array}{l}
f^{\prime} \\
h^{\prime}
\end{array}\right]\right\} \in \widetilde{A}\right\} .
$$

The proof of Proposition 2.2 is contained in [19, Theorem 3.9]. Moreover, it is shown in [19] that the converse is also true, every Nevanlinna family generates via (2.2) the Weyl family of a symmetric linear operator $S$. In the case when the pair $\{\varphi, \psi\}$ is equivalent to a pair $\left\{I_{\mathcal{L}}, m\right\}$ with $m \in N[\mathcal{L}]$, functional model $A(m)$ for this symmetric operator $S$ was given in [6] (see also [17]).

In the following theorem we give another functional model of a selfadjoint linear relation $\widetilde{A}$ recovered from a Nevanlinna pair. Consider the reproducing kernel Hilbert space $\mathcal{H}(\Phi, \Psi)$, which is characterized by the properties:

(1) $\mathrm{N}_{\omega}^{\Phi \Psi}(\lambda) u \in \mathcal{H}(\Phi, \Psi)$ for all $\omega \in \mathbb{C} \backslash \mathbb{R}$ and $u \in \mathcal{L}$;

(2) for every $f \in \mathcal{H}(\Phi, \Psi)$ the following identity holds

$$
\left\langle f(\cdot), \mathbf{N}_{\omega}^{\Phi \Psi}(\lambda) u\right\rangle_{\mathcal{H}(\Phi, \Psi)}=(f(\omega), u)_{\mathcal{L}}, \quad \omega \in \mathbb{C} \backslash \mathbb{R}, u \in \mathcal{L} .
$$

It follows from (2.6) that the evaluation operator $E(\lambda): f \mapsto f(\lambda)(f \in \mathcal{H}(\Phi, \Psi))$ is a bounded operator from $\mathcal{H}(\Phi, \Psi)$ to $\mathcal{L}$.

Theorem 2.5. Let $\{\Phi, \Psi\} \in \tilde{N}(\mathcal{L})$. Then the linear relation

$$
\widetilde{A}(\Phi, \Psi)=\left\{\left\{\left[\begin{array}{l}
f \\
u
\end{array}\right],\left[\begin{array}{l}
f^{\prime} \\
u^{\prime}
\end{array}\right]\right\}: \begin{array}{c}
f, f^{\prime} \in \mathcal{H}(\Phi, \Psi), u, u^{\prime} \in \mathcal{L}, \\
f^{\prime}(\lambda)-\lambda f(\lambda)=\Phi(\bar{\lambda})^{*} u-\Psi(\bar{\lambda})^{*} u^{\prime}
\end{array}\right\}
$$

is a selfadjoint linear relation in $\mathcal{H}(\Phi, \Psi) \oplus \mathcal{L}$ and the normalized pair $\{\varphi, \psi\}$ given by (2.1) is the Nevanlinna pair corresponding to $\widetilde{A}(\Phi, \Psi)$ and $\mathcal{L}$.

Proof. Step 1. Let us show that $\widetilde{A}(\Phi, \Psi)$ contains vectors of the form

$$
\left\{F_{\omega} u, F_{\omega}^{\prime} u\right\}:=\left\{\left[\begin{array}{c}
\mathrm{N}_{\omega}(\cdot) u \\
\Psi(\bar{\omega})
\end{array}\right],\left[\begin{array}{c}
\bar{\omega} \mathrm{N}_{\omega}(\cdot) u \\
\Phi(\bar{\omega}) u
\end{array}\right]\right\}, \quad u \in \mathcal{L}, \omega \in \mathbb{C}_{+} \cup \mathbb{C}_{-},
$$


where $\mathrm{N}_{\omega}(\cdot)=\mathrm{N}_{\omega}^{\Phi \Psi}(\cdot)$ and the restriction $\widetilde{A}^{\prime}$ of $\widetilde{A}(\Phi, \Psi)$ to the span of vectors $\left\{F_{\omega} u, F_{\omega}^{\prime} u\right\}$ is a symmetric linear relation.

Indeed, it follows from (2.7) and the equality

$$
(\bar{\omega}-\lambda) \mathrm{N}_{\omega}(\lambda) u=\Phi(\bar{\lambda})^{*} \Psi(\bar{\omega})-\Psi(\bar{\lambda})^{*} \Phi(\bar{\omega})
$$

that $\left\{F_{\omega} u, F_{\omega}^{\prime} u\right\} \in \widetilde{A}(\Phi, \Psi)$.

For arbitrary $\omega_{j} \in \mathbb{C} \backslash \mathbb{R}, u_{j} \in \mathcal{L}(j=1,2)$ one obtains

$$
\begin{aligned}
\left\langle\bar{\omega}_{1}\right. & \left.\mathrm{N}_{\omega_{1}}(\cdot) u_{1}, \mathrm{~N}_{\omega_{2}}(\cdot) u_{2}\right\rangle_{\mathcal{H}(\Phi, \Psi)}-\left\langle\mathrm{N}_{\omega_{1}}(\cdot) u_{1}, \bar{\omega}_{2} \mathrm{~N}_{\omega_{2}}(\cdot) u_{2}\right\rangle_{\mathcal{H}(\Phi, \Psi)} \\
& +\left(\Phi\left(\bar{\omega}_{1}\right) u_{1}, \Psi\left(\bar{\omega}_{2}\right) u_{2}\right)_{\mathcal{L}}-\left(\Psi\left(\bar{\omega}_{1}\right) u_{1}, \Phi\left(\bar{\omega}_{2}\right) u_{2}\right)_{\mathcal{L}} \\
& =\left(\bar{\omega}_{1}-\omega_{2}\right)\left(\mathrm{N}_{\omega_{1}}\left(\omega_{2}\right) u_{1}, u_{2}\right)_{\mathcal{L}}-\left(\left(\Phi\left(\bar{\omega}_{2}\right)^{*} \Psi\left(\bar{\omega}_{1}\right)-\Psi\left(\bar{\omega}_{2}\right)^{*} \Phi\left(\bar{\omega}_{1}\right)\right) u_{1}, u_{2}\right)_{\mathcal{L}} \\
& =0
\end{aligned}
$$

therefore, $\widetilde{A}^{\prime}$ is symmetric in $\mathcal{H}(\Phi, \Psi) \oplus \mathcal{L}$.

Step 2. Let us show that $\operatorname{ran}\left(\widetilde{A}^{\prime}-\lambda\right)$ is dense in $\mathcal{H}(\Phi, \Psi) \oplus \mathcal{L}$ for $\lambda \in \mathbb{C} \backslash \mathbb{R}$. Choose the vector $\left\{F_{\omega} u, F_{\omega}^{\prime} u\right\}$ with $\omega=\bar{\lambda}$. Then

$$
\left\{\left[\begin{array}{l}
\mathrm{N}_{\bar{\lambda}}(\cdot) u \\
\Psi(\lambda) u
\end{array}\right],\left[\begin{array}{c}
0 \\
\Phi(\lambda) u-\lambda \Psi(\lambda) u
\end{array}\right]\right\} \in \widetilde{A}^{\prime}-\lambda .
$$

Since $\operatorname{ran}(\Phi(\lambda) u-\lambda \Psi(\lambda))=\mathcal{L}$ one obtains $0 \oplus \mathcal{L} \subset \operatorname{ran}\left(\widetilde{A}^{\prime}-\lambda\right)$. Taking $\widehat{F}_{\omega} u$ with $\omega \neq \bar{\lambda}$ one obtains from (2.8)

$$
\left\{\left[\begin{array}{c}
\mathrm{N}_{\omega}(\cdot) u \\
\Psi(\bar{\omega}) u
\end{array}\right],\left[\begin{array}{c}
(\bar{\omega}-\lambda) \mathrm{N}_{\omega}(\cdot) u \\
\Phi(\bar{\omega}) u-\lambda \Psi(\bar{\omega}) u
\end{array}\right]\right\} \in \widetilde{A}^{\prime}-\lambda
$$

and, hence, $\left[\begin{array}{c}\mathrm{N}_{\omega}(\cdot) u \\ 0\end{array}\right] \in \operatorname{ran}\left(\widetilde{A}^{\prime}-\lambda\right)$ for all $\omega \neq \bar{\lambda}$. Due to the properties 1) and 2) of $\mathcal{H}(\Phi, \Psi)$ one obtains the statement.

Step 3. Let us show that $\widetilde{A}(\Phi, \Psi)=\left(\widetilde{A}^{\prime}\right)^{*}$. Indeed, for every vector

$$
\widehat{F}=\left\{F, F^{\prime}\right\}=\left\{\left[\begin{array}{c}
f(\cdot) \\
u
\end{array}\right],\left[\begin{array}{c}
f^{\prime}(\cdot) \\
u^{\prime}
\end{array}\right]\right\} \in \widetilde{A}(\Phi, \Psi), \quad f, f^{\prime} \in \mathcal{H}(\Phi, \Psi), u, u^{\prime} \in \mathcal{L},
$$

and $\omega \in \mathbb{C} \backslash \mathbb{R}, v \in \mathcal{L}$ it follows from (2.7) that

$$
\begin{aligned}
\left\langle F^{\prime}, F_{\omega} v\right\rangle_{\mathcal{H}(\Phi, \Psi)}-\left\langle F, F_{\omega}^{\prime} v\right\rangle_{\mathcal{H}(\Phi, \Psi)} & =\left\langle f^{\prime}, \mathrm{N}_{\omega}(\cdot) v\right\rangle_{\mathcal{H}(\Phi, \Psi)}-\left\langle f, \bar{\omega} \mathrm{N}_{\omega}(\cdot) v\right\rangle_{\mathcal{H}(\Phi, \Psi)} \\
& +\left(u^{\prime}, \Psi(\bar{\omega}) v\right)_{\mathcal{L}}-(u, \Phi(\bar{\omega}) v)_{\mathcal{L}} \\
& =\left(f^{\prime}(\omega)-\omega f(\omega)+\Psi(\bar{\omega})^{*} u^{\prime}-\Phi(\bar{\omega})^{*} u, v\right)_{\mathcal{L}} \\
& =0
\end{aligned}
$$

Hence $\widehat{F} \in\left(\widetilde{A}^{\prime}\right)^{*}$ and $\widetilde{A}(\Phi, \Psi) \subset\left(\widetilde{A}^{\prime}\right)^{*}$.

Conversely, if

$$
\left\langle f^{\prime}, \mathrm{N}_{\omega}(\cdot) h\right\rangle_{\mathcal{H}(\Phi, \Psi)}-\left\langle f, \bar{\omega} \mathbf{N}_{\omega}(\cdot) v\right\rangle_{\mathcal{H}(\Phi, \Psi)}+\left(u^{\prime}, \Psi(\bar{\omega}) v\right)_{\mathcal{L}}-(u, \Phi(\bar{\omega}) v)_{\mathcal{L}}=0
$$

for some $f, f^{\prime} \in \mathcal{H}(\varphi, \psi), u, u^{\prime} \in \mathcal{L}$ and all $\omega \in \mathbb{C} \backslash \mathbb{R}, v \in \mathcal{L}$, then

$$
f^{\prime}(\omega)-\omega f(\omega)-\left(\Phi(\bar{\omega})^{*} u-\Psi(\bar{\omega})^{*} u^{\prime}\right)=0
$$


and, hence, $\widehat{F}=\left\{\left[\begin{array}{c}f(\cdot) \\ u\end{array}\right],\left[\begin{array}{c}f^{\prime}(\cdot) \\ u^{\prime}\end{array}\right]\right\} \in \widetilde{A}(\Phi, \Psi)$. This proves that $\left(\widetilde{A}^{\prime}\right)^{*} \subset$ $\widetilde{A}(\Phi, \Psi)$.

Step 4. And finally, in view of (2.9) and the property (ii) of Definition 2.1] one obtains

$$
\begin{aligned}
\psi(\lambda) & =\left.P_{\mathcal{L}}(\widetilde{A}(\Phi, \Psi)-\lambda)^{-1}\right|_{\mathcal{L}}=\Psi(\lambda)(\Phi(\lambda)-\lambda \Psi(\lambda))^{-1}, \\
\varphi(\lambda) & =I_{\mathcal{L}}+\lambda \psi(\lambda)=\Phi(\lambda)(\Phi(\lambda)-\lambda \Psi(\lambda))^{-1}
\end{aligned}
$$

Therefore, the pair $\{\varphi, \psi\}$ is a normalized Nevanlinna pair corresponding to the linear relation $\widetilde{A}(\Phi, \Psi)$ and the scale $\mathcal{L}$.

Remark 2.6. In notations of [19] the pair $\{\Phi, \Psi\}$ generates via (2.2) the Weyl family of the symmetric operator

$$
S(\Phi, \Psi)=\left\{\left\{f, f^{\prime}\right\}: f, f^{\prime} \in \mathcal{H}(\Phi, \Psi), f^{\prime}(\lambda)-\lambda f(\lambda)=0\right\}
$$

corresponding to the boundary relation

$$
\Gamma=\left\{\left\{\left[\begin{array}{c}
f \\
f^{\prime}
\end{array}\right],\left[\begin{array}{l}
u^{\prime} \\
u
\end{array}\right]\right\}: \begin{array}{c}
f, f^{\prime} \in \mathcal{H}(\Phi, \Psi), u, u^{\prime} \in \mathcal{L} \\
f^{\prime}(\lambda)-\lambda f(\lambda)=\Phi(\bar{\lambda})^{*} u-\Psi(\bar{\lambda})^{*} u^{\prime}
\end{array}\right\} .
$$

Remark 2.7. Mention that the linear space

$$
\mathfrak{N}_{\bar{\omega}}(T):=\left\{\mathrm{N}_{\omega}(\cdot) u: u \in \mathcal{L}\right\}
$$

in general is not closed, since

$$
\begin{aligned}
\left(\mathrm{N}_{\omega}(\cdot) u, \mathrm{~N}_{\omega}(\cdot) u\right)_{\mathcal{H}(\varphi, \psi)} & =\left(\mathrm{N}_{\omega}(\omega) u, u\right)_{\mathcal{L}} \\
& =\left(\frac{\Phi(\bar{\omega})^{*} \Psi(\bar{\omega})-\Psi(\bar{\omega})^{*} \Phi(\omega)}{\omega-\bar{\omega}} u, u\right)
\end{aligned}
$$

and the operator $\mathrm{N}_{\omega}(\omega)$ not necessarily is boundedly invertible. If, however, $0 \in$ $\rho\left(\mathrm{N}_{\omega}(\omega)\right)$ then $\mathfrak{N}_{\bar{\omega}}(T)$ is closed. Recall that in this case $0 \in \rho\left(\mathrm{N}_{\lambda}(\lambda)\right)$ for all $\lambda \in \mathbb{C} \backslash \mathbb{R}$ and, hence, all the subspace $\mathfrak{N}_{\lambda}(\bar{\lambda})$ are closed.

Let, the ovf $\gamma(\lambda): \mathcal{L} \rightarrow \mathcal{H}$ be defined by

$$
\gamma(\lambda):=\left.P_{\mathcal{H}}(\widetilde{A}-\lambda)^{-1}\right|_{\mathcal{L}} \quad(\lambda \in \rho(\widetilde{A})) .
$$

Proposition 2.8. Let $\widetilde{A}$ be a selfadjoint linear relation in $\mathcal{H} \oplus \mathcal{L}$ and let $\{\varphi, \psi\}$ be the normalized Nevanlinna pair given by (2.3). Then the following identity holds

$$
\mathrm{N}_{\omega}^{\varphi \psi}(\lambda)=\gamma(\bar{\lambda})^{*} \gamma(\bar{\omega}) \text {. }
$$

Proof. Indeed, it follows from (2.5) that the kernel $\mathrm{N}_{\omega}^{\varphi \psi}(\lambda)$ for the normalized Nevanlinna pair takes the form

$$
\mathrm{N}_{\omega}^{\varphi \psi}(\lambda)=\left(P_{\mathcal{L}} R_{\lambda} P_{\mathcal{H}}\right)\left(\left.P_{\mathcal{H}} R_{\bar{\omega}}\right|_{\mathcal{L}}\right)=\gamma(\bar{\lambda})^{*} \gamma(\bar{\omega}) .
$$

In general case one obtains

$$
\begin{aligned}
\mathrm{N}_{\omega}^{\Phi \Psi}(\lambda) & =(\Phi(\bar{\lambda})-\lambda \Psi(\bar{\lambda}))^{*} \mathrm{~N}_{\omega}^{\varphi \psi}(\lambda)(\Phi(\bar{\omega})-\bar{\omega} \Psi(\bar{\omega})) \\
& =(\Phi(\bar{\lambda})-\lambda \Psi(\bar{\lambda}))^{*} \gamma(\bar{\lambda})^{*} \gamma(\bar{\omega})(\Phi(\bar{\omega})-\bar{\omega} \Psi(\bar{\omega})) .
\end{aligned}
$$

The following statement formulated in terms of boundary relations can be found in [18, Lemma 4.1] 
Lemma 2.9. Let $\widetilde{A}$ be a selfadjoint linear relation in $\mathcal{H} \oplus \mathcal{L}$, let $\{\varphi, \psi\}$ be the normalized Nevanlinna pair given by (2.3) and lel $\operatorname{dim} \mathcal{L}<\infty$. Then:

(i) $\operatorname{ker} \psi(\lambda)=0$ for $\lambda \in \mathbb{C} \backslash \mathbb{R}$ iff $P_{\mathcal{L}} \operatorname{dom} \widetilde{A}$ is dense in $\mathcal{L}$;

(ii) $\operatorname{ker} \varphi(\lambda)=0$ for $\lambda \in \mathbb{C} \backslash \mathbb{R}$ iff $P_{\mathcal{L}} \operatorname{ran} \widetilde{A}$ is dense in $\mathcal{L}$.

Proof. Let us prove the first statement. The set $P_{\mathcal{L}} \operatorname{dom} \widetilde{A}$ consists of the vectors $u \in \mathcal{L}$ such that

$$
\left\{\left[\begin{array}{l}
f \\
u
\end{array}\right],\left[\begin{array}{l}
f^{\prime} \\
u^{\prime}
\end{array}\right]\right\} \in \widetilde{A} \quad \text { for some } \quad f, f^{\prime} \in \mathcal{H}, u^{\prime} \in \mathcal{L} .
$$

If there is a vector $v \in \mathcal{L}$ such that $v \perp u$ for all $u \in P_{\mathcal{L}} \operatorname{dom} \widetilde{A}$ then

$$
\left\{\left[\begin{array}{l}
0 \\
0
\end{array}\right],\left[\begin{array}{l}
0 \\
v
\end{array}\right]\right\} \in \widetilde{A}
$$

and then $\psi(\lambda) v=0, \varphi(\lambda) v=v$, due to (2.3).

Conversely, if $\psi(\lambda) v=0$ for some $v \neq 0$, then in view of (2.3)

$$
\left\{\left[\begin{array}{l}
0 \\
0
\end{array}\right],\left[\begin{array}{l}
0 \\
v
\end{array}\right]\right\} \in \widetilde{A}
$$

and hence $v \perp P_{\mathcal{L}} \operatorname{dom} \widetilde{A}$.

The proof of the second statement is similar.

If the Nevanlinna pair $\{\varphi, \psi\}$ satisfies the first condition (i) in Lemma 2.9 then it is equivalent to a Nevanlinna function $m \in N(\mathcal{L})$. If, additionally, $m(\lambda)$ takes values in $[\mathcal{L}]$ for $\lambda \in \mathbb{C} \backslash \mathbb{R}$ then the reproducing kernel Hilbert space $\mathcal{H}(\varphi, \psi)$ is unitary equivalent to the reproducing kernel Hilbert space $\mathcal{H}(m)$ with the kernel $\mathrm{N}_{\omega}^{m}(\lambda)$ (see (1.1)) via the mapping

$$
\mathcal{U}: f \in \mathcal{H}(m) \rightarrow(I-\lambda m(\lambda))^{-1} f(\lambda) \in \mathcal{H}(\varphi, \psi) .
$$

These spaces have been introduced by L. de Branges [13]. The following statement can be derived from [5], however we will give a proof for the convenience of the reader.

Lemma 2.10. Let $m \in N[\mathcal{L}]$, and let $\operatorname{dim} \mathcal{L}<\infty$. Then:

(i) $f(\lambda)=O(1)(\lambda \widehat{\rightarrow} \infty)$ for all $f \in \mathcal{H}(m)$;

(ii) If, additionally, $m \in N_{0}[\mathcal{L}]$ then $f(\lambda)=O\left(\frac{1}{\lambda}\right)(\lambda \widehat{\rightarrow} \infty)$ for all $f \in \mathcal{H}(m)$.

Proof. It follows from the reproducing kernel property and Schwartz inequality that

$$
\begin{aligned}
\left|(f(\lambda), u)_{\mathcal{L}}\right| & =\left|\left\langle f(\cdot), \mathrm{N}_{\lambda}(\cdot) u\right\rangle_{\mathcal{H}(m)}\right| \\
& \leq\|f(\cdot)\|_{\mathcal{H}(m)}|| \mathrm{N}_{\lambda}(\cdot) u \|_{\mathcal{H}(m)} \\
& =\|f\|_{\mathcal{H}(m)}\left(\frac{\Im m(\lambda)}{\Im \lambda} u, u\right)^{1 / 2}=O(1)
\end{aligned}
$$

for all $f \in \mathcal{H}(m)$ and $u \in \mathcal{L}$. If, additionally, $m \in N_{0}[\mathcal{L}]$, then

$$
\left(\frac{\Im m(\lambda)}{\Im \lambda} u, u\right)=\int \frac{d(\sigma(t) u, u)}{|t-\lambda|^{2}}=O\left(\frac{1}{\lambda^{2}}\right)
$$


for some finite measure $d \sigma$ and, hence,

$$
(f(\lambda), u)_{\mathcal{L}}=O\left(\frac{1}{\lambda}\right)
$$

\subsection{Generalized Fourier transform.}

Definition 2.11. A selfadjoint linear relation $\widetilde{A}$ in $\mathcal{H} \oplus \mathcal{L}$ is said to be $\mathcal{L}$-minimal if

$$
\mathcal{H}_{0}=\overline{\operatorname{span}}\left\{P_{\mathcal{H}}(\widetilde{A}-\lambda)^{-1} \mathcal{L}: \lambda \in \rho(\widetilde{A})\right\} .
$$

In this section we show that every $\mathcal{L}$-minimal selfadjoint linear relation $A$ is unitarily equivalent to its functional model $A(\varphi, \psi)$, constructed in Theorem 2.5. The operator $\mathcal{F}: \mathcal{H} \rightarrow \mathcal{H}(\varphi, \psi)$ given by

$$
h \mapsto(\mathcal{F} h)(\lambda)=\gamma(\bar{\lambda})^{*} h=P_{\mathcal{L}}(\widetilde{A}-\lambda)^{-1} h \quad(h \in \mathcal{H})
$$

is called the generalized Fourier transform associated with $\widetilde{A}$ and the scale $\mathcal{L}$.

Theorem 2.12. let $\widetilde{A}$ be a selfadjoint linear relation in $\mathcal{H} \oplus \mathcal{L}$ and let $\{\varphi, \psi\}$ be the corresponding Nevanlinna pair given by (2.3). Then:

1) The generalized Fourier transform $\mathcal{F}$ maps isometrically the subspace $\mathcal{H}_{0}$ onto $\mathcal{H}(\varphi, \psi)$ and $\mathcal{F}$ is identically equal to 0 on $\mathcal{H} \ominus \mathcal{H}_{0}$.

2) For every $\left\{\left[\begin{array}{l}f \\ u\end{array}\right],\left[\begin{array}{l}f^{\prime} \\ u^{\prime}\end{array}\right]\right\} \in \widetilde{A}$ the following identity holds

$$
E(\lambda) \mathcal{F}\left(f^{\prime}-\lambda f\right)=[\varphi(\lambda)-\psi(\lambda)]\left[\begin{array}{c}
u \\
u^{\prime}
\end{array}\right]
$$

Proof. 1) For every vector $h=\gamma(\bar{\omega}) u(\omega \in \rho(\widetilde{A}), u \in \mathcal{L})$ it follows from Proposition 2.8 that

$$
(\mathcal{F} h)(\lambda)=\gamma(\bar{\lambda})^{*} \gamma(\bar{\omega}) u=\mathrm{N}_{\omega}^{\varphi \psi}(\lambda) u .
$$

Therefore, $\mathcal{F}$ maps the linear space $\operatorname{span}\{\gamma(\omega) \mathcal{L}: \omega \in \rho(\widetilde{A})\}$ dense in $\mathcal{H}_{0}$ onto the linear space $\operatorname{span}\left\{\mathrm{N}_{\omega}^{\varphi \psi}(\cdot) \mathcal{L}: \omega \in \rho(\widetilde{A})\right\}$ which is dense in $\mathcal{H}(\varphi, \psi)$. Moreover, this mapping is isometric, since

$$
\begin{aligned}
(\mathcal{F} h, \mathcal{F} h)_{\mathcal{H}(\varphi, \psi)} & =\left(\mathrm{N}_{\omega}^{\varphi \psi}(\cdot) u, \mathrm{~N}_{\omega}^{\varphi \psi}(\cdot) u\right)_{\mathcal{H}(\varphi, \psi)} \\
& =\left(\mathbf{N}_{\omega}^{\varphi \psi}(\omega) u, u\right)_{\mathcal{L}}=(h, h)_{\mathcal{H}} .
\end{aligned}
$$

This proves the first statement. It is clear from (2.14) that $\mathcal{F} h \equiv 0$ for $h \in \mathcal{H} \ominus \mathcal{H}_{0}$.

2) Let

$$
g=\gamma(\bar{\omega}) v=P_{\mathcal{H}}(\widetilde{A}-\bar{\omega})^{-1} v, \quad v \in \mathcal{L}
$$

Then it follows from (2.3), (2.11) that

$$
\left[\begin{array}{c}
g \\
\psi(\bar{\omega}) v
\end{array}\right]=(\widetilde{A}-\bar{\omega})^{-1}\left[\begin{array}{l}
0 \\
v
\end{array}\right], \quad\left[\begin{array}{c}
\bar{\omega} g \\
\varphi(\bar{\omega}) v
\end{array}\right]=\left[I+\bar{\omega}(\widetilde{A}-\bar{\omega})^{-1}\right]\left[\begin{array}{l}
0 \\
v
\end{array}\right]
$$

and hence

$$
\left\{\left[\begin{array}{c}
g \\
\psi(\bar{\omega}) v
\end{array}\right],\left[\begin{array}{c}
\bar{\omega} g \\
\varphi(\bar{\omega}) v
\end{array}\right]\right\} \in \widetilde{A}
$$


Since $\widetilde{A}=\widetilde{A}^{*}$ one obtains for all $\left\{\left[\begin{array}{l}f \\ u\end{array}\right],\left[\begin{array}{l}f^{\prime} \\ u^{\prime}\end{array}\right]\right\} \in \widetilde{A}$

$$
\left(f^{\prime}, g\right)_{\mathcal{H}}-(f, \bar{\omega} g)_{\mathcal{H}}+\left(u^{\prime}, \psi(\bar{\omega})\right)_{\mathcal{L}}-(u, \varphi(\bar{\omega}) v)_{\mathcal{L}}=0, \quad v \in \mathcal{L}
$$

This implies

$$
\gamma(\bar{\omega})^{*}\left(f^{\prime}-\bar{\omega} f\right)=\varphi(\omega) u-\psi(\omega) u^{\prime}, \quad \omega \in \mathbb{C} \backslash \mathbb{R} .
$$

This proves the identity (2.15).

Remark 2.13. In the case, when the linear relation $\widetilde{A}$ is $\mathcal{L}$-minimal it is unitary equivalent to the linear relation $A(\varphi, \psi)$ via the formula

$$
\widetilde{A}(\varphi, \psi)=\left\{\left\{\left[\begin{array}{c}
\mathcal{F} f \\
u
\end{array}\right],\left[\begin{array}{c}
\mathcal{F} f^{\prime} \\
u^{\prime}
\end{array}\right]\right\}:\left\{\left[\begin{array}{l}
f \\
u
\end{array}\right],\left[\begin{array}{l}
f^{\prime} \\
u^{\prime}
\end{array}\right]\right\} \in \widetilde{A}\right\} .
$$

The operator $\mathcal{F} \oplus I_{\mathcal{L}}$ establishes this unitary equivalence.

\section{Abstract interpolation problem}

Let $\mathcal{X}$ be a complex linear space, let $\mathcal{L}$ be a Hilbert space, let $B_{1}, B_{2}$ be linear operators in $\mathcal{X}$, let $C_{1}, C_{2}$ be linear operators from $\mathcal{X}$ to $\mathcal{H}$, and let $K$ be a nonegative sesquilinear form on $\mathcal{X}$ which satisfies the following identity

(A1) $K\left(B_{2} h, B_{1} g\right)-K\left(B_{1} h, B_{2} g\right)=\left(C_{1} h, C_{2} g\right)_{\mathcal{L}}-\left(C_{2} h, C_{1} g\right)_{\mathcal{L}}$

for every $h, g \in \mathcal{X}$. Consider the following

Problem $\operatorname{AIP}\left(B_{1}, B_{2}, C_{1}, C_{2}, K\right)$. Let the data set $\left(B_{1}, B_{2}, C_{1}, C_{2}, K\right)$ satisfies the assumption $(A 1)$. Find a normalized Nevanlinna pair $\{\varphi, \psi\} \in \widetilde{N}(\mathcal{L})$ such that for some linear mapping $F: \mathcal{X} \rightarrow \mathcal{H}(\varphi, \psi)$ the following conditions hold:

$(\mathrm{C} 1)\left(F B_{2} h\right)(\lambda)-\lambda\left(F B_{1} h\right)(\lambda)=[\varphi(\lambda)-\psi(\lambda)]\left[\begin{array}{l}C_{1} h \\ C_{2} h\end{array}\right]$;

(C2) $\|F h\|_{\mathcal{H}(\varphi, \psi)}^{2} \leq K(h, h)$

for all $h \in \mathcal{X}$.

Clearly ker $K=\{h \in \mathcal{X}: K(h, h)=0\}$ is a linear subspace of $\mathcal{X}$. Consider the factor space $\widehat{\mathcal{X}}=\mathcal{X} /$ ker $K$ and denote by $\hat{h}$ the equivalence class $h+\operatorname{ker} K$ in $\widehat{\mathcal{X}}$, $h \in \mathcal{X}$. Let $\widehat{\mathcal{X}}$ be endowed with the scalar product

$$
(\widehat{h}, \widehat{g})_{\mathcal{H}}=K(h, g), \quad h, g \in \mathcal{X}
$$

and let $\mathcal{H}$ be the completion of $\widehat{\mathcal{X}}$ with respect to the norm $\|h\|_{\mathcal{H}}$.

In examples (see Section 5 ) the linear space $\mathcal{X}$ has an original inner product. In order to avoid an ambiguity we denote by $B^{+}$the adjoint to the operator $B: \mathcal{H} \rightarrow \mathcal{H}$ and by $B^{*}$ the adjoint to $B: \mathcal{X} \rightarrow \mathcal{X}$.

Proposition 3.1. Let the data set $\left(B_{1}, B_{2}, C_{1}, C_{2}, K\right)$ satisfies the assumption $(A 1)$. Then the linear relation

$$
\widehat{A}=\left\{\left\{\left[\begin{array}{l}
\widehat{B_{1} h} \\
C_{1} h
\end{array}\right],\left[\begin{array}{l}
\widehat{B_{2} h} \\
C_{2} h
\end{array}\right]\right\}: h \in \mathcal{X}\right\}
$$

is symmetric in $\mathcal{H} \oplus \mathcal{L}$. 
Proof. The statement is implied by (3.2) since

$$
\begin{aligned}
& \left\langle\left[\begin{array}{l}
\widehat{B_{2} h} \\
C_{2} h
\end{array}\right],\left[\begin{array}{l}
\widehat{B_{1} h} \\
C_{1} h
\end{array}\right]\right\rangle_{\mathcal{H} \oplus \mathcal{L}}-\left\langle\left[\begin{array}{l}
\widehat{B_{1} h} \\
C_{1} h
\end{array}\right],\left[\begin{array}{l}
\widehat{B_{2} h} \\
C_{2} h
\end{array}\right]\right\rangle_{\mathcal{H} \oplus \mathcal{L}} \\
& =K\left(B_{2} h, B_{1} h\right)-K\left(B_{1} h, B_{2} h\right)-\left(C_{1} h, C_{2} h\right)_{\mathcal{L}}+\left(C_{2} h, C_{1} h\right)_{\mathcal{L}}=0 .
\end{aligned}
$$

Remark 3.2. In general, the linear relation $\widehat{A}$ need not be simple and its deficiency indices not necessarily coincide.

Theorem 3.3. Let the data set $\left(B_{1}, B_{2}, C_{1}, C_{2}, K\right)$ satisfies the assumption $(A 1)$. Then the Problem $\operatorname{AIP}\left(B_{1}, B_{2}, C_{1}, C_{2}, K\right)$ is solvable and the set of its normalized solutions is parametrized by the formula

$$
\left[\begin{array}{c}
\psi(\lambda) \\
\varphi(\lambda)
\end{array}\right]=\left[\begin{array}{cc}
I_{\mathcal{L}} & 0 \\
\lambda & I_{\mathcal{L}}
\end{array}\right]\left[\begin{array}{c}
\left.P_{\mathcal{L}}(\widetilde{A}-\lambda)^{-1}\right|_{\mathcal{L}} \\
I_{\mathcal{L}}
\end{array}\right],
$$

where $\widetilde{A}$ ranges over the set of all selfadjoint extensions of $\widehat{A}$ with the exit in a Hilbert space $\widetilde{\mathcal{H}} \oplus \mathcal{L} \supset \mathcal{H} \oplus \mathcal{L}$. The corresponding linear mapping $F: \mathcal{X} \rightarrow \mathcal{H}(\varphi, \psi)$ is given by

$$
(F h)(\lambda)=P_{\mathcal{L}}(\widetilde{A}-\lambda)^{-1} \widehat{h}, \quad h \in \mathcal{X} .
$$

Proof. Sufficiency. Let $\widetilde{A}$ be a selfadjoint extension of $\widehat{A}$ and let $\{\varphi, \psi\}$ be the normalized Nevanlinna pair corresponding to $\widetilde{A}$ and the scale $\mathcal{L}$, and let $\mathcal{F}: \widetilde{\mathcal{H}} \rightarrow$ $\mathcal{H}(\varphi, \psi)$ be the corresponding generalized Fourier transform given by (2.14). Then the formula (3.3) is implied by (2.3) and in view of (2.14) the linear mapping $F$ : $\mathcal{X} \rightarrow \mathcal{H}(\varphi, \psi)$ given by (3.4) is connected to $\mathcal{F}$ via the formula

$$
F h=\mathcal{F} \widehat{h}, \quad(h \in \mathcal{X}) .
$$

Since $\mathcal{F}$ satisfies the identity $(2.15)$ and

$$
\left\{\left[\begin{array}{l}
\widehat{B_{1} h} \\
C_{1} h
\end{array}\right],\left[\begin{array}{l}
\widehat{B_{2} h} \\
C_{2} h
\end{array}\right]\right\} \in A \subset \widetilde{A}
$$

one obtains from (2.15)

$$
\begin{aligned}
\left(F B_{2} h\right)(\lambda)-\lambda\left(F B_{1} h\right)(\lambda) & =\left(\mathcal{F} \widehat{B_{2} h}\right)(\lambda)-\lambda\left(\mathcal{F} \widehat{B_{1} h}\right)(\lambda) \\
& =\left[\begin{array}{ll}
\varphi(\lambda) & -\psi(\lambda)
\end{array}\right]\left[\begin{array}{c}
C_{1} h \\
C_{2} h
\end{array}\right] \quad \forall h \in \mathcal{H} .
\end{aligned}
$$

Next, it follows from (3.1) and Theorem 2.12 that

$$
\|F h\|_{\mathcal{H}(\varphi, \psi)}^{2}=\|\mathcal{F} \widehat{h}\|_{\mathcal{H}(\varphi, \psi)}^{2} \leq\|\widehat{h}\|_{\mathcal{H}}^{2}=K(h, h)
$$

This proves $(C 1),(C 2)$ and, hence, $\{\varphi, \psi\}$ is a solution of the $A I P$.

Necessity. Let a normalized Nevanlinna pair $\{\varphi, \psi\}$ be a solution of the AIP and let the mapping $F: \mathcal{X} \rightarrow \mathcal{H}(\varphi, \psi)$ satisfies (C1), (C2). We will construct a selfadjoint exit space extension $\widetilde{A}$ of $\widehat{A}$ such that (3.3) and (3.4) hold.

Step 1. Isometric embedding of $\mathcal{H}$ into a Hilbert space. It follows from $(C 2)$ that

$$
(F h)(\lambda) \equiv 0 \text { for all } h \in \operatorname{ker} K \text {. }
$$


Thus $F$ induces the mapping $\widehat{F}: \widehat{\mathcal{X}} \rightarrow \mathcal{H}(\varphi, \psi)$, which is well defined by the equality

$$
\hat{h} \rightarrow(\widehat{F h})(\lambda)=(F h)(\lambda), \quad h \in \mathcal{H}
$$

and is contractive due to $(C 2)$

$$
\|(\widehat{F} \widehat{h})(\lambda)\|_{\mathcal{H}(\varphi, \psi)}^{2}=\|(F h)(\lambda)\|_{\mathcal{H}(\varphi, \psi)}^{2} \leq K(h, h)=\|\widehat{h}\|_{\mathcal{H}}^{2} .
$$

We will keep the same notation for the continuous extension of $\widehat{F}$ to $\mathcal{H}$.

Let $D=D^{*}(\geq 0)$ be the defect operator of the contraction $\widehat{F}$ defined by

$$
D^{2}=I-\widehat{F}^{+} \widehat{F}: \mathcal{H} \rightarrow \mathcal{H}
$$

and let $\mathcal{D}=\overline{\operatorname{ran}} D$ be the defect subspace of $\widehat{F}$ in $\mathcal{H}$. Consider the column extension $\widetilde{F}$ of the operator $\widehat{F}$ to the isometric mapping from $\mathcal{H}$ to $\mathcal{D} \oplus \mathcal{H}(\varphi, \psi)$ by the formula

$$
\widetilde{F} h=\left[\begin{array}{c}
D h \\
\widehat{F} h
\end{array}\right], \quad h \in \mathcal{H}
$$

Step 2. Construction of a selfadjoint linear relation $\widetilde{A}$. Let $A_{\mathcal{D}}$ be a linear relation in $\mathcal{D}$ defined by

$$
A_{\mathcal{D}}=\left\{\left\{D \widehat{B}_{1} h, D \widehat{B}_{2} h\right\}: h \in \mathcal{X}\right\}
$$

and let us show that $A_{\mathcal{D}}$ is symmetric in $\mathcal{D}$. Indeed, it follows from (3.8), (3.9) that

$$
\begin{aligned}
\left(D \widehat{B_{2} h}, D \widehat{B_{1} h}\right)_{\mathcal{H}} & -\left(D \widehat{B_{1} h}, D \widehat{B_{2} h}\right)_{\mathcal{H}} \\
& =\left(\left(I-\widehat{F}^{+} \widehat{F}\right) \widehat{B_{2} h}, \widehat{B_{1} h}\right)_{\mathcal{H}}-\left((I-\widehat{F}+\widehat{F}) \widehat{B_{1} h}, \widehat{B_{2} h}\right)_{\mathcal{H}} \\
& =K\left(B_{2} h, B_{1} h\right)-K\left(B_{1} h, B_{2} h\right) \\
& -\left(\widehat{F} \widehat{B_{2} h}, \widehat{F} \widehat{B_{1} h}\right)_{\mathcal{H}(\varphi, \psi)}+\left(\widehat{F} \widehat{B_{1} h}, \widehat{F} \widehat{B_{2} h}\right)_{\mathcal{H}(\varphi, \psi)} .
\end{aligned}
$$

As follows from $(\mathrm{C} 1)$

$$
\begin{aligned}
\left(\widehat{F} \widehat{B_{2} h}\right)(\lambda)-\lambda\left(\widehat{F} \widehat{B_{1} h}\right)(\lambda) & =\left(F B_{2} h\right)(\lambda)-\lambda\left(F B_{1} h\right)(\lambda) \\
& =\left[\begin{array}{ll}
\varphi(\lambda) & \psi(\lambda)
\end{array}\right]\left[\begin{array}{l}
C_{1} h \\
C_{2} h
\end{array}\right] \quad \forall h \in \mathcal{X}
\end{aligned}
$$

In view of (2.7) this implies

$$
\left\{\left[\begin{array}{c}
\widehat{F} \widehat{B_{1} h} \\
C_{1} h
\end{array}\right],\left[\begin{array}{c}
\widehat{F} \widehat{B_{2} h} \\
C_{2} h
\end{array}\right]\right\} \in A(\varphi, \psi) .
$$

Therefore, the right hand part of (3.11) can be rewritten as

$$
K\left(B_{2} h, B_{1} h\right)-K\left(B_{1} h, B_{2} h\right)-\left(C_{1} h, C_{2} h\right)_{\mathcal{L}}+\left(C_{2} h, C_{1} h\right)_{\mathcal{L}}
$$

and hence it is vanishing due to (A1).

Let $\widetilde{A}_{\mathcal{D}}$ be a selfadjoint extension of $A_{\mathcal{D}}$ in a Hilbert space $\widetilde{\mathcal{D}} \supset \mathcal{D}$ and let $\widetilde{A}=$ $\widetilde{A}_{\mathcal{D}} \oplus A(\varphi, \psi)$.

Step 3. Linear relation $\widetilde{A}$ satisfies (3.3) and (3.4). Under the identification of the vector $h \in \mathcal{H}$ with $\widetilde{F} h$ the symmetric linear relation $\widehat{A}$ in $\mathcal{H} \oplus \mathcal{L}$ can be identified 
with the symmetric linear relation

$$
\begin{aligned}
& A_{1}=\left(\widetilde{F} \oplus I_{\mathcal{L}}\right) \widehat{A}\left(\widetilde{F} \oplus I_{\mathcal{L}}\right)^{-1} \\
& =\left\{\left\{\left[\begin{array}{c}
D \widehat{B_{1} h} \\
\widehat{F} \widehat{B_{1} h} \\
C_{1} h
\end{array}\right],\left[\begin{array}{c}
D \widehat{B_{2} h} \\
\widehat{F} \widehat{B_{2} h} \\
C_{2} h
\end{array}\right]\right\}: h \in \mathcal{X}\right\}
\end{aligned}
$$

in $\widetilde{\mathcal{H}}:=\widetilde{\mathcal{D}} \oplus \mathcal{H}(\varphi, \psi) \oplus \mathcal{L}$. Moreover $A_{1}$ is contained in the selfadjoint linear relation

$$
\widetilde{A}=\widetilde{A}_{\mathcal{D}} \oplus A(\varphi, \psi)
$$

since $\left\{D \widehat{B_{1} h}, D \widehat{B_{2} h}\right\} \in A_{\mathcal{D}} \subset \widetilde{A_{\mathcal{D}}}$ and

$$
\left\{\left[\begin{array}{c}
\widehat{F} \widehat{B_{1} h} \\
C_{1} h
\end{array}\right],\left[\begin{array}{c}
\widehat{F} \widehat{B_{2} h} \\
C_{2} h
\end{array}\right]\right\} \in A(\varphi, \psi) .
$$

The formula (3.3) is implied by the analogous formula for $A(\varphi, \psi)$

$$
\left[\begin{array}{c}
\psi(\lambda) \\
\varphi(\lambda)
\end{array}\right]=\left[\begin{array}{cc}
I_{\mathcal{L}} & 0 \\
\lambda I_{\mathcal{L}} & I_{\mathcal{L}}
\end{array}\right]\left[\begin{array}{c}
\left.P_{\mathcal{L}}(A(\varphi, \psi)-\lambda)^{-1}\right|_{\mathcal{L}} \\
I_{\mathcal{L}}
\end{array}\right]
$$

since

$$
\left.P_{\mathcal{L}}(A(\varphi, \psi)-\lambda)^{-1}\right|_{\mathcal{L}}=\left.P_{\mathcal{L}}(\widetilde{A}-\lambda)^{-1}\right|_{\mathcal{L}}
$$

Moreover, for every $h \in \mathcal{X}$ one obtains

$$
\begin{aligned}
P_{\mathcal{L}}(\widetilde{A}-\lambda)^{-1}\left[\begin{array}{c}
\widetilde{F} \widehat{h} \\
0
\end{array}\right] & =P_{\mathcal{L}}(\widetilde{A}-\lambda)^{-1}\left[\begin{array}{c}
\widehat{F} h \\
0
\end{array}\right] \\
& =P_{\mathcal{L}}(A(\varphi, \psi)-\lambda)^{-1}\left[\begin{array}{c}
F h \\
0
\end{array}\right] \\
& =\mathcal{F}(\varphi, \psi) F h=F h .
\end{aligned}
$$

This proves the formula (3.4), since $\widetilde{F} \widehat{h}$ is identified with $\widehat{h}$.

Remark 3.4. If $\left(B_{1}, B_{2}, C_{1}, C_{2}, K\right)$ is a data set for the AIP in Nevanlinna classes then the data set $\left(T_{1}, T_{2}, M_{1}, M_{2}, K\right)$ given by

$$
\begin{gathered}
T_{1}=B_{1}-i B_{2}, \quad T_{2}=B_{1}+i B_{2} ; \\
M_{1}=C_{1}-i C_{2}, \quad M_{2}=C_{1}+i C_{2},
\end{gathered}
$$

is a data set for the AIP in the Schur class. Recall (see [25]), that a contractive $[\mathcal{L}]$ valued function $\omega(\zeta)$ is said to be a solution of the problem $A I P\left(T_{1}, T_{2}, M_{1}, M_{2}, K\right)$, if there exists a map $\Phi$ from $\mathcal{X}$ to the de Branges Rovnyak space $\mathcal{H}_{\omega}$, such that

$$
\left(\Phi T_{1} h\right)(t)-t\left(\Phi T_{2} h\right)(t)=\left[\begin{array}{cc}
I & -\omega(t) \\
-\omega^{*}(t) & I
\end{array}\right]\left[\begin{array}{l}
M_{2} h \\
M_{1} h
\end{array}\right]
$$

and $\|\Phi h\|_{\mathcal{H}_{\omega}}^{2} \leq K(h, h)$ for all $h \in \mathcal{X}$. One can check, that solutions of the problems $A I P\left(B_{1}, B_{2}, C_{1}, C_{2}, K\right)$ and $A I P\left(T_{1}, T_{2}, M_{1}, M_{2}, K\right)$ are related via some linear fractional transformation and the result of Theorem 3.3 can be derived from the corresponding result in [25]. However, we prefer to give a direct proof based on the de Branges Rovnyak space $\mathcal{H}(\varphi, \psi)$, especially since these spaces will be usefull in applications to some interpolation problems. 
In general, the mapping $F: \mathcal{X} \rightarrow \mathcal{H}(\varphi, \psi)$ in (C1), (C2) is not uniquely defined by the solution $\{\varphi, \psi\}$ of the abstract interpolation problem $A I P\left(B_{1}, B_{2}, C_{1}, C_{2}, K\right)$. We impose an additional assumption on the data set:

(U) $B_{2}-\lambda B_{1}$ is an isomorphism in $\mathcal{X}$ for $\lambda$ in nonempty domains $\mathcal{O}_{ \pm} \subset \mathbb{C}_{ \pm}$, which ensures the uniqueness of $F$. Let us set

$$
G(\lambda)=\left[\begin{array}{l}
C_{1} \\
C_{2}
\end{array}\right]\left(B_{2}-\lambda B_{1}\right)^{-1} \quad\left(\lambda \in \mathcal{O}_{ \pm}\right)
$$

Proposition 3.5. Let the data set $\left(B_{1}, B_{2}, C_{1}, C_{2}, K\right)$ satisfies the assumptions (A1), (U). Then the mapping $F: \mathcal{X} \rightarrow \mathcal{H}(\varphi, \psi)$ in (C1), (C2) is uniquely defined by the solution $\{\varphi, \psi\}$ of the abstract interpolation problem $A I P\left(B_{1}, B_{2}, C_{1}, C_{2}, K\right)$ by the formula

$$
(F h)(\lambda)=[\varphi(\lambda)-\psi(\lambda)] G(\lambda) h \quad\left(\lambda \in \mathcal{O}_{ \pm}\right) .
$$

Proof. Applying (C1) to the vector

$$
h=h_{\mu}:=\left(B_{2}-\mu B_{1}\right)^{-1} g \quad\left(\mu \in \mathcal{O}_{ \pm}, g \in \mathcal{X}\right),
$$

one obtains

$$
\begin{aligned}
(F g)(\lambda) & =\left(F B_{2} h_{\mu}\right)(\lambda)-\mu\left(F B_{1} h_{\mu}\right)(\lambda) \\
& =\left(F B_{2} h_{\mu}\right)(\lambda)-\lambda\left(F B_{1} h_{\mu}\right)(\lambda)+(\lambda-\mu)\left(F B_{1} h_{\mu}\right)(\lambda) \\
& =[\varphi(\lambda)-\psi(\lambda)] G(\mu) g+(\lambda-\mu)\left(F B_{1} h_{\mu}\right)(\lambda) .
\end{aligned}
$$

Setting in (3.14) $\lambda=\mu$, one obtains

$$
(F g)(\mu))=[\varphi(\mu)-\psi(\mu)] G(\mu) g \quad\left(\mu \in \mathcal{O}_{ \pm}, g \in \mathcal{X}\right)
$$

\section{Description of SOlutions of ABStract interpolation PROBlem.}

In view of Theorem 3.3 a description of the set of solutions of the AIP is reduced to the description of $\mathcal{L}$-resolvents of the linear relation $\widehat{A}$. The later problem can be solved within the theory of $\mathcal{L}$-resolvent matrix [30, 33. In this section we will treat both the nondegenerate (ker $K \neq\{0\}$ ) and the degenerate case (ker $K \neq\{0\}$ ). In the case when the form $K(\cdot, \cdot)$ is degenerate it will be more convenient to calculate the resolvent matrix of some auxiliary linear relation $A_{0}$ which is a restriction of $\widehat{A}$.

4.1. Symmetric linear relation $A$ and boundary triplet for $A^{+}$. In this section we impose some additional assumptions on the data set $\left(B_{1}, B_{2}, C_{1}, C_{2}, K\right)$.

(A2) $\operatorname{dim} \operatorname{ker} K<\infty$ and $\mathcal{X}$ admits the representation

$$
\mathcal{X}=\mathcal{X}_{0} \dot{+} \operatorname{ker} K
$$

such that $B_{j} \mathcal{X}_{0} \subseteq \mathcal{X}_{0}(j=1,2)$.

(A3) $B_{2}=I_{\mathcal{X}}$ and the operators $\left.B_{1}\right|_{\mathcal{X}_{0}}: \mathcal{X}_{0} \subseteq \mathcal{H} \rightarrow \mathcal{H},\left.C_{1}\right|_{\mathcal{X}_{0}},\left.C_{2}\right|_{\mathcal{X}_{0}}: \mathcal{X}_{0} \subseteq \mathcal{H} \rightarrow \mathcal{L}$ are bounded. 
Due to the assumption (A2) one can identify $\mathcal{X}_{0}$ with $\widehat{\mathcal{X}}=\mathcal{X} /$ ker $K$ and consider the space $\mathcal{H}$ as a completion of $\mathcal{X}_{0}$. The continuations of the operators $\left.B_{1}\right|_{\mathcal{X}_{0}},\left.C_{1}\right|_{\mathcal{X}_{0}}$, $\left.C_{2}\right|_{\mathcal{X}_{0}}$ will be denoted by $\widetilde{B}_{1} \in[\mathcal{H}], \widetilde{C}_{1}, \widetilde{C}_{2} \in[\mathcal{H}, \mathcal{L}]$.

Define a linear relation

$$
A_{0}=\left\{\left\{\left[\begin{array}{l}
B_{1} h \\
C_{1} h
\end{array}\right],\left[\begin{array}{l}
B_{2} h \\
C_{2} h
\end{array}\right]\right\}: h \in \mathcal{X}_{0}\right\}
$$

in a Hilbert space $\mathcal{H} \oplus \mathcal{L}$. Clearly, $A_{0}$ is a restriction of the symmetric linear relation $\widehat{A}$ which can be rewritten as

$$
\widehat{A}=\left\{\left\{\left[\begin{array}{c}
B_{1} h+\widehat{B_{1} u} \\
C_{1} h+C_{1} u
\end{array}\right],\left[\begin{array}{c}
B_{2} h+\widehat{B_{2} u} \\
C_{2} h+C_{2} u
\end{array}\right]\right\}: h \in \mathcal{X}_{0}, u \in \operatorname{ker} K\right\} .
$$

In view of (A3) the closures of $A_{0}$ and $\widehat{A}$ take the form

$$
\begin{gathered}
A:=\operatorname{clos} A_{0}=\left\{\left\{\left[\begin{array}{c}
\widetilde{B}_{1} h \\
\widetilde{C}_{1} h
\end{array}\right],\left[\begin{array}{c}
h \\
\widetilde{C}_{2} h
\end{array}\right]\right\}: h \in \mathcal{H}\right\}, \\
\operatorname{clos} \widehat{A}=\left\{\left\{\left[\begin{array}{c}
\widetilde{B}_{1} h+\widehat{B_{1} u} \\
\widetilde{C}_{1} h+C_{1} u
\end{array}\right],\left[\begin{array}{c}
h \\
\widetilde{C}_{2} h+C_{2} u
\end{array}\right]\right\}: h \in \mathcal{H}, u \in \operatorname{ker} K\right\} .
\end{gathered}
$$

Recall the definition of the boundary triplet for nondensely defined symmetric operator.

Definition 4.1. [22], [35] A triplet $\Pi=\left\{\mathcal{L}, \Gamma_{1}, \Gamma_{2}\right\}$, where $\Gamma_{i}: \widehat{A}^{+} \rightarrow \mathcal{L}, i=0,1$, is said to be a boundary triplet for $\widehat{A}^{+}$, if for all $\widehat{f}=\left\{f, f^{\prime}\right\}, \widehat{g}=\left\{g, g^{\prime}\right\} \in \widehat{A}^{+}$;

$$
\left(f^{\prime}, g\right)_{\mathcal{H} \oplus \mathcal{L}}-\left(f, g^{\prime}\right)_{\mathcal{H} \oplus \mathcal{L}}=\left(\Gamma_{1} \widehat{f}, \Gamma_{2} \widehat{g}\right)_{\mathcal{L}}-\left(\Gamma_{2} \widehat{f}, \Gamma_{1} \widehat{g}\right)_{\mathcal{L}}
$$

and the mapping $\Gamma:=\left[\begin{array}{l}\Gamma_{1} \\ \Gamma_{0}\end{array}\right]: \widehat{A}^{+} \rightarrow\left[\begin{array}{l}\mathcal{L} \\ \mathcal{L}\end{array}\right]$ is surjective.

A point $\lambda \in \mathbb{C}$ is said to be a regular type point for a closed symmetric linear relation $A$ if $\operatorname{ran}(A-\lambda)$ is closed in $\mathcal{H} \oplus \mathcal{L}$. It is well known that the set $\widehat{\rho}(A)$ of regular type points for symmetric linear relation $A$ contains $\mathbb{C}_{+} \cup \mathbb{C}_{-}$and the defect subspaces

$$
\mathfrak{N}_{\lambda}(A):=(\mathcal{H} \oplus \mathcal{L}) \ominus \operatorname{ran}(A-\bar{\lambda})
$$

have the same dimensions $n_{+}(A)$ and $n_{-}(A)$ for $\lambda \in \mathbb{C}_{+}$and $\lambda \in \mathbb{C}_{-}$, respectively. The numbers $n_{+}(A)$ and $n_{-}(A)$ are called the defect numbers of the symmetric linear relation $A$. In the following proposition we show, that the symmetric linear relation $A$ in (4.4) has equal defect numbers $n_{+}(A)=n_{-}(A)=\operatorname{dim} \mathcal{L}$ and, moreover, $0 \in \widehat{\rho}(A)$.

Proposition 4.2. Let the data set $\left(B_{1}, B_{2}, C_{1}, C_{2}, K\right)$ satisfy the assumptions (A1)-(A3). Then:

(1) the adjoint linear relation $A^{+}$takes the form

$$
A^{+}=\left\{\widehat{g}=\left\{\left[\begin{array}{l}
g \\
v
\end{array}\right],\left[\begin{array}{c}
g^{\prime} \\
v^{\prime}
\end{array}\right]\right\}: \begin{array}{c}
v, v^{\prime} \in \mathcal{L}, g^{\prime} \in \mathcal{H} \\
g=\widetilde{B}_{1}^{+} g^{\prime}+\widetilde{C}_{1}^{+} v^{\prime}-\widetilde{C}_{2}^{+} v
\end{array}\right\}
$$


(2) the set $\widehat{\rho}(A)$ of regular type points for symmetric linear relation $A$ contains the resolvent set of the linear relation $\widetilde{B}_{1}^{-1}$

$$
\rho\left(\widetilde{B}_{1}^{-1}\right)=\left\{\lambda \in(\mathbb{C} \backslash\{0\}): 1 / \lambda \in \rho\left(\widetilde{B}_{1}\right)\right\} \cup\{0\} \text { if } \widetilde{B}_{1} \in[\mathcal{H}]
$$

and the defect subspace $\mathfrak{N}_{\lambda}(A)$ for $\lambda \in \rho\left(\widetilde{B}_{1}^{-1}\right)$ consists of the vectors

$$
\left[\begin{array}{c}
-F(\bar{\lambda})^{+} u \\
u
\end{array}\right], \quad u \in \mathcal{L}
$$

where $F(\lambda)=\left(\widetilde{C}_{2}-\lambda \widetilde{C}_{1}\right)\left(I_{\mathcal{H}}-\lambda \widetilde{B}_{1}\right)^{-1}$;

(3) a boundary triplet $\Pi=\left\{\mathcal{L}, \Gamma_{1}, \Gamma_{2}\right\}$ for $\widehat{A}^{+}$can be defined by

$$
\Gamma_{1} \widehat{g}=-v+\widetilde{C}_{1} g^{\prime}, \quad \Gamma_{2} \widehat{g}=v^{\prime}-\widetilde{C}_{2} g^{\prime} .
$$

Proof. 1) Let

$$
\widehat{g}=\left\{\left[\begin{array}{l}
g \\
v
\end{array}\right],\left[\begin{array}{l}
g^{\prime} \\
v^{\prime}
\end{array}\right]\right\} \in A^{+} \quad\left(g, g^{\prime} \in \mathcal{H} ; v, v^{\prime} \in \mathcal{L}\right) .
$$

Then it follows from (4.4) that

$$
\left(g^{\prime}, \widetilde{B}_{1} h\right)_{\mathcal{H}}-(g, h)_{\mathcal{H}}+\left(v^{\prime}, \widetilde{C}_{1} h\right)_{\mathcal{L}}-\left(v, \widetilde{C}_{2} h\right)_{\mathcal{L}}=0
$$

for all $h \in \mathcal{H}$ and, therefore,

$$
\widetilde{B}_{1}^{+} g^{\prime}-g+\widetilde{C}_{1}^{+} v^{\prime}-\widetilde{C}_{2}^{+} v=0 .
$$

This gives the equality

$$
g=\widetilde{B}_{1}^{+} g^{\prime}+\widetilde{C}_{1}^{+} v^{\prime}-\widetilde{C}_{2}^{+} v .
$$

2) It follows from (4.4) that

$$
A-\lambda=\left\{\left\{\left[\begin{array}{c}
\widetilde{B}_{1} h \\
\widetilde{C}_{1} h
\end{array}\right],\left[\begin{array}{l}
\left(I_{\mathcal{H}}-\lambda \widetilde{B}_{1}\right) h \\
\left(\widetilde{C}_{2}-\lambda \widetilde{C}_{1}\right) h
\end{array}\right]\right\}: h \in \mathcal{H}\right\},
$$

and, hence

$$
\operatorname{ran}(A-\lambda)=\left\{\left[\begin{array}{c}
h \\
F(\lambda) h
\end{array}\right]: h \in \mathcal{H}\right\}
$$

where $F(\lambda)=\left(\widetilde{C}_{2}-\lambda \widetilde{C}_{1}\right)\left(I_{\mathcal{H}}-\lambda \widetilde{B}_{1}\right)^{-1}$. Therefore, ran $(A-\lambda)$ is closed for all $\lambda \in \rho\left(\widetilde{B}_{1}^{-1}\right)$.

If $\lambda \in \rho\left(\widetilde{B}_{1}^{-1}\right)$ and $\widehat{g} \in \widehat{\mathfrak{N}}_{\lambda}(\widehat{A})$ then $g^{\prime}=\lambda g, v^{\prime}=\lambda v$. Substituting these equalities in (4.9) one obtains

$$
\left(I_{\mathcal{H}}-\lambda \widetilde{B}_{1}^{+}\right) g=-\left(\widetilde{C}_{2}^{+}-\lambda \widetilde{C}_{1}^{+}\right) v
$$

This proves the second statement since

$$
g=-F(\bar{\lambda})^{+} v
$$

3) For two vectors

$$
\widehat{f}=\left\{\left[\begin{array}{l}
f \\
v
\end{array}\right],\left[\begin{array}{l}
f^{\prime} \\
v^{\prime}
\end{array}\right]\right\}, \quad \widehat{g}=\left\{\left[\begin{array}{l}
g \\
v
\end{array}\right],\left[\begin{array}{l}
g^{\prime} \\
v^{\prime}
\end{array}\right]\right\} \in A^{+}
$$


one obtains

$$
\begin{aligned}
\left(f^{\prime}, g\right)_{\mathcal{H}} & -\left(f, g^{\prime}\right)_{\mathcal{H}}+\left(u^{\prime}, v\right)_{\mathcal{L}}-\left(u, v^{\prime}\right)_{\mathcal{L}}=\left(u^{\prime}, v\right)_{\mathcal{L}}-\left(u, v^{\prime}\right)_{\mathcal{L}} \\
& +\left(f^{\prime}, \widetilde{B}_{1}^{+} g^{\prime}+\widetilde{C}_{1}^{+} v^{\prime}-\widetilde{C}_{2}^{+} v\right)_{\mathcal{H}}-\left(\widetilde{B}_{1}^{+} f^{\prime}+\widetilde{C}_{1}^{+} u-\widetilde{C}_{2}^{+} u^{\prime}, g^{\prime}\right)_{\mathcal{H}} .
\end{aligned}
$$

Then the right hand part of (4.11) takes the form

$$
\begin{aligned}
\left(\widetilde{B}_{1} f^{\prime}, g^{\prime}\right)_{\mathcal{H}} & -\left(f^{\prime}, \widetilde{B}_{1} g^{\prime}\right)_{\mathcal{H}}+\left(\widetilde{C}_{1} f^{\prime}, v^{\prime}\right)_{\mathcal{L}}-\left(\widetilde{C}_{2} f^{\prime}, v\right)_{\mathcal{L}} \\
& -\left(u^{\prime}, \widetilde{C}_{1} g^{\prime}\right)_{\mathcal{L}}+\left(u, \widetilde{C}_{2} g^{\prime}\right)_{\mathcal{L}}+\left(u^{\prime}, v\right)_{\mathcal{L}}-\left(u, v^{\prime}\right)_{\mathcal{L}} \\
& =\left(\widetilde{C}_{2} f^{\prime}, \widetilde{C}_{1} g^{\prime}\right)_{\mathcal{L}}-\left(\widetilde{C}_{1} f^{\prime}, \widetilde{C}_{2} g^{\prime}\right)_{\mathcal{L}}+\left(\widetilde{C}_{1} f^{\prime}, v^{\prime}\right)_{\mathcal{L}}-\left(\widetilde{C}_{2} f^{\prime}, v\right)_{\mathcal{L}} \\
& -\left(u^{\prime}, \widetilde{C}_{1} g^{\prime}\right)_{\mathcal{L}}+\left(u, \widetilde{C}_{2} g^{\prime}\right)_{\mathcal{L}}+\left(u^{\prime}, v\right)_{\mathcal{L}}-\left(u, v^{\prime}\right)_{\mathcal{L}} \\
& =\left(\widetilde{C}_{2} f^{\prime}-u^{\prime}, \widetilde{C}_{1} g^{\prime}-v\right)_{\mathcal{L}}-\left(\widetilde{C}_{1} f^{\prime}-u, \widetilde{C}_{2} g^{\prime}-v^{\prime}\right)_{\mathcal{L}}
\end{aligned}
$$

Clearly, the mapping $\Gamma: A^{+} \rightarrow \mathcal{L} \oplus \mathcal{L}$ is surjective and hence the triplet $\left\{\mathcal{L}, \Gamma_{1}, \Gamma_{2}\right\}$ is a boundary triplet for $A^{+}$.

4.2. $\mathcal{L}$-resolvent matrix. Recall some facts from M.G. Krein s representation theory ([31], [17]) for symmetric linear relation $A$ in $\mathcal{H} \oplus \mathcal{L}$. Let us say that $\lambda \in \rho(A, \mathcal{L})$ if $\lambda$ is a regular type point for $A$ and

$$
\mathcal{H} \oplus \mathcal{L}=\operatorname{ran}(A-\lambda) \dot{+} \mathcal{L}
$$

For every $\lambda \in \rho(A, \mathcal{L})$ the operator valued function $\mathcal{P}(\lambda): \mathcal{H} \rightarrow \mathcal{L}$ is defined as a skew projection onto $\mathcal{L}$ in the decomposition (4.12) and $\mathcal{Q}(\lambda): \mathcal{H} \rightarrow \mathcal{L}$ is given by

$$
\mathcal{Q}(\lambda)=P_{\mathcal{L}}(A-\lambda)^{-1}(I-\mathcal{P}(\lambda)), \quad \lambda \in \rho(A, \mathcal{L}) .
$$

Let the operator-valued functions $\widehat{\mathcal{P}}(\lambda)^{+}$and $\widehat{\mathcal{Q}}(\lambda)^{+}$be given by

$$
\begin{gathered}
\widehat{\mathcal{P}}(\lambda)^{+} u=\left\{\mathcal{P}(\lambda)^{+} u, \bar{\lambda} \mathcal{P}(\lambda)^{+} u\right\}, \quad u \in \mathcal{L}, \\
\widehat{\mathcal{Q}}(\lambda)^{+} u=\left\{\mathcal{Q}(\lambda)^{+} u, u+\bar{\lambda} \mathcal{Q}(\lambda)^{+} u\right\}, \quad u \in \mathcal{L},
\end{gathered}
$$

where $\widehat{\mathcal{P}}(\lambda)^{+}, \widehat{\mathcal{Q}}(\lambda)^{+}: \mathcal{L} \rightarrow \mathcal{H}$ are adjoint operators to $\mathcal{P}(\lambda), \mathcal{Q}(\lambda): \mathcal{H} \rightarrow \mathcal{L}$. The role of the ovf's $\mathcal{P}(\lambda), \mathcal{Q}(\lambda)$ is clear from the following theorem.

Theorem 4.3. [30, 33, 17]. Let $\Pi=\left\{\mathcal{L}, \Gamma_{1}, \Gamma_{2}\right\}$ be a boundary triplet for $A^{+}$. The set of $\mathcal{L}$-resolvents of $A$ is parametrized by the formula

$$
\left.P_{\mathcal{L}}(\widetilde{A}-\lambda)^{-1}\right|_{\mathcal{L}}=\left(w_{11}(\lambda) q(\lambda)+w_{12}(\lambda) p(\lambda)\right)\left(w_{21}(\lambda) q(\lambda)+w_{22}(\lambda) p(\lambda)\right)^{-1}
$$

where $(p, q)$ ranges over the set $\tilde{\mathcal{N}}(\mathcal{L})$ of Nevanlinna pairs and the block matrix $W(\lambda)=\left(w_{i j}(\lambda)\right)_{i, i=1}^{2}$ is given by

$$
W_{\Pi \mathcal{L}}(\lambda)=\left[\begin{array}{ll}
-\Gamma_{2} \widehat{\mathcal{Q}}(\lambda)^{*} & \Gamma_{2} \widehat{\mathcal{P}}(\lambda)^{*} \\
-\Gamma_{1} \widehat{\mathcal{Q}}(\lambda)^{*} & \Gamma_{1} \widehat{\mathcal{Q}}(\lambda)^{*}
\end{array}\right]^{*} \quad \lambda \in \rho(\widehat{A}, \mathcal{L}) .
$$

In the following Theorem we calculate all the objects of M.G.Krein representation theory $\mathcal{P}(\lambda), \mathcal{Q}(\lambda)$ and the $\mathcal{L}$-resolvent matrix $W_{\Pi \mathcal{L}}(\lambda)$ for the linear relation $A$ in (4.4).

Theorem 4.4. Let $B_{1}, B_{2}, C_{1}, C_{2}, K$ satisfy the assumptions $(A 1)-(A 3)$. Then: 
(1) $\rho(A, \mathcal{L})=\rho\left(B_{1}^{-1}\right)$ and for $\lambda \in \rho(A, \mathcal{L})$, the ovf's $\mathcal{P}(\lambda), \mathcal{Q}(\lambda)$ are given by

$$
\begin{gathered}
\mathcal{P}(\lambda)\left[\begin{array}{l}
f \\
u
\end{array}\right]=u-F(\lambda) f, \quad f \in \mathcal{H}, \quad u \in \mathcal{L}, \\
\mathcal{Q}(\lambda)\left[\begin{array}{l}
f \\
u
\end{array}\right]=\widetilde{C}_{1}\left(I_{\mathcal{H}}-\lambda \widetilde{B}_{1}\right)^{-1} f, \quad f \in \mathcal{H} ;
\end{gathered}
$$

(2) The adjoint operators to $\mathcal{P}(\lambda), \mathcal{Q}(\lambda):\left[\begin{array}{l}\mathcal{H} \\ \mathcal{L}\end{array}\right] \rightarrow \mathcal{L}$ take the form

$$
\begin{gathered}
\mathcal{P}(\lambda)^{+} u=\left[\begin{array}{c}
-F(\lambda)^{+} u \\
u
\end{array}\right] \quad u \in \mathcal{L}, \lambda \in \rho(A, \mathcal{L}), \\
\mathcal{Q}(\lambda)^{+} u=\left[\begin{array}{c}
\left(I_{\mathcal{H}}-\bar{\lambda} \widetilde{B}_{1}^{+}\right)^{-1} \widetilde{C}_{1}^{+} u \\
0
\end{array}\right] \quad u \in \mathcal{L}, \lambda \in \rho(A, \mathcal{L}) ;
\end{gathered}
$$

(3) The $\mathcal{L}$-resolvent matrix $W_{\Pi \mathcal{L}}(\lambda)$ corresponding to the boundary triplet $\Pi$ takes the form

$$
W_{\Pi \mathcal{L}}(\lambda)=\left[\begin{array}{cc}
-I_{\mathcal{L}} & 0 \\
\lambda & -I_{\mathcal{L}}
\end{array}\right]\left(I_{\mathcal{L} \oplus \mathcal{L}}+i \lambda\left[\begin{array}{c}
\widetilde{C}_{1} \\
\widetilde{C}_{2}
\end{array}\right]\left(I_{\mathcal{H}}-\lambda \widetilde{B}_{1}\right)^{-1}\left[\begin{array}{c}
\widetilde{C}_{1} \\
\widetilde{C}_{2}
\end{array}\right]^{+} J\right) .
$$

Proof. 1) Assume that $\lambda \in \rho(A, \mathcal{L})$ and the decomposition (4.12) holds. Then for $f \in \mathcal{H}, u \in \mathcal{L}$ there are unique $h \in \mathcal{H}$ and $v \in \mathcal{L}$ such that

$$
\left(I_{\mathcal{H}}-\lambda \widetilde{B}_{1}\right) h=f, \quad\left(\widetilde{C}_{2}-\lambda \widetilde{C}_{1}\right) h+v=u .
$$

This implies, in particular, that $\lambda \in \rho\left(B_{1}^{-1}\right)$. Conversely, if $\lambda \in \rho\left(B_{1}^{-1}\right)$, then the equations (4.23) have unique solutions $h \in \mathcal{H}$ and $v \in \mathcal{L}$ and, hence, $\lambda \in \rho(A, \mathcal{L})$. In view of (4.23) these solutions take the form

$$
h=\left(I_{\mathcal{H}}-\lambda \widetilde{B}_{1}\right)^{-1} f, \quad v=\mathcal{P}(\lambda)\left[\begin{array}{l}
f \\
u
\end{array}\right]=u-F(\lambda) f .
$$

It follows from (4.13), (4.24) and (4.10) that

$$
\begin{aligned}
\mathcal{Q}(\lambda)\left[\begin{array}{l}
f \\
u
\end{array}\right] & =P_{\mathcal{L}}(A-\lambda)^{-1}\left[\begin{array}{c}
f \\
F(\lambda) f
\end{array}\right]=P_{\mathcal{L}}\left[\begin{array}{c}
\widetilde{B}_{1} h \\
\widetilde{C}_{1} h
\end{array}\right] \\
& =\widetilde{C}_{1}\left(I_{\mathcal{H}}-\lambda \widetilde{B}_{1}\right)^{-1} f .
\end{aligned}
$$

2) The formulas (4.20), (4.21) are implied by

$$
\begin{aligned}
\left\langle\mathcal{P}(\lambda)^{+} v,\left[\begin{array}{c}
f \\
u
\end{array}\right]\right\rangle_{\mathcal{H} \oplus \mathcal{L}} & =(v, u-F(\lambda) f)_{\mathcal{L}} \\
& =\left\langle\left[\begin{array}{c}
-F(\lambda)^{+} v \\
v
\end{array}\right],\left[\begin{array}{l}
f \\
u
\end{array}\right]\right\rangle_{\mathcal{H} \oplus \mathcal{L}} \\
\left\langle\mathcal{Q}(\lambda)^{+} v,\left[\begin{array}{c}
f \\
u
\end{array}\right]\right\rangle_{\mathcal{H} \oplus \mathcal{L}} & =\left(v, \widetilde{C}_{1}\left(I_{\mathcal{H}}-\lambda \widetilde{B}_{1}\right)^{-1} f\right)_{\mathcal{L}} \\
& =\left\langle\left[\begin{array}{c}
\left(I_{\mathcal{H}}-\lambda \widetilde{B}_{1}^{+}\right)^{-1} \widetilde{C}_{1}^{+} v \\
0
\end{array}\right],\left[\begin{array}{c}
f \\
u
\end{array}\right]\right\rangle_{\mathcal{H} \oplus \mathcal{L}} .
\end{aligned}
$$


Now one obtains from (4.14), (4.20) and (4.8) that

$$
\begin{aligned}
& \Gamma_{2} \widehat{\mathcal{P}}(\lambda)^{+} v=\bar{\lambda} v+\bar{\lambda} \widetilde{C}_{2} F(\lambda)^{+} v, \\
& \Gamma_{1} \widehat{\mathcal{P}}(\lambda)^{+} v=-v-\bar{\lambda} \widetilde{C}_{1} F(\lambda)^{+} v .
\end{aligned}
$$

Similarly (4.15), (4.21) and (4.8) imply

$$
\begin{gathered}
\Gamma_{2} \widehat{\mathcal{Q}}(\lambda)^{+} v=v-\bar{\lambda} \widetilde{C}_{2}\left(I_{\mathcal{H}}-\lambda \widetilde{B}_{1}^{+}\right)^{-1} \widetilde{C}_{1}^{+} v \\
\Gamma_{1} \widehat{\mathcal{Q}}(\lambda)^{+} v=\bar{\lambda} \widetilde{C}_{1}\left(I_{\mathcal{H}}-\lambda \widetilde{B}_{1}^{+}\right)^{-1} \widetilde{C}_{1}^{+} v
\end{gathered}
$$

It follows from (4.25)- (4.28) and (4.17) that

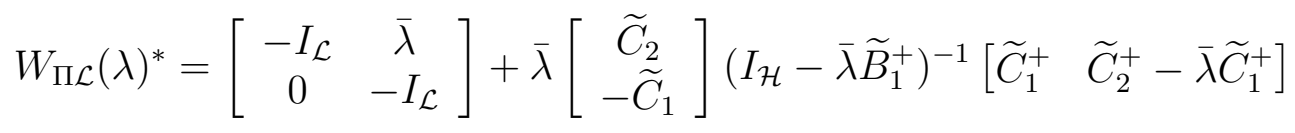

and hence

$$
\begin{aligned}
W_{\Pi \mathcal{L}}(\lambda) & =\left[\begin{array}{cc}
-I & 0 \\
\lambda & -I
\end{array}\right]+\lambda\left[\begin{array}{c}
\widetilde{C}_{1} \\
\widetilde{C}_{2}-\lambda \widetilde{C}_{1}
\end{array}\right]\left(I_{\mathcal{H}}-\lambda \widetilde{B}_{1}\right)^{-1}\left[\widetilde{C}_{2}^{+}-\widetilde{C}_{1}^{+}\right] \\
& =\left[\begin{array}{cc}
-I & 0 \\
\lambda & -I
\end{array}\right]\left(I_{\mathcal{L} \oplus \mathcal{L}}-\lambda\left[\begin{array}{c}
\widetilde{C}_{1} \\
\widetilde{C}_{2}
\end{array}\right]\left(I_{\mathcal{H}}-\lambda \widetilde{B}_{1}\right)^{-1}\left[\widetilde{C}_{2}^{+}-\widetilde{C}_{1}^{+}\right]\right) .
\end{aligned}
$$

Corollary 4.5. Let the data set $\left(B_{1}, B_{2}, C_{1}, C_{2}, K\right)$ satisfy $(A 1),(A 2)$ and

$\left(A 3^{\prime}\right)$ the operators $\left.B_{1}\right|_{\mathcal{X}_{0}},\left.B_{2}\right|_{\mathcal{X}_{0}}: \mathcal{X}_{0} \subset \mathcal{H} \rightarrow \mathcal{H},\left.C_{1}\right|_{\mathcal{X}_{0}},\left.C_{2}\right|_{\mathcal{X}_{0}}: \mathcal{X}_{0} \subset \mathcal{H} \rightarrow \mathcal{L}$ are bounded, $\left(B_{2}-\mu B_{1}\right) \mathcal{X}=\mathcal{X}$ for some $\mu \in \mathbb{R}$, and the continuations $\widetilde{B}_{1}, \widetilde{B}_{2}$ of the operators $\left.B_{1}\right|_{\mathcal{X}_{0}},\left.B_{2}\right|_{\mathcal{X}_{0}}$ satisfy the condition $0 \in \rho\left(\widetilde{B}_{2}-\mu \widetilde{B}_{1}\right)$.

Then one of the $\mathcal{L}$-resolvent matrices can be found from

$$
\begin{aligned}
& {\left[\begin{array}{cc}
-I & 0 \\
\lambda & -I
\end{array}\right]^{-1} W^{\mu}(\lambda)} \\
& =I+i(\lambda-\mu)\left[\begin{array}{l}
\widetilde{C}_{1} \\
\widetilde{C}_{2}
\end{array}\right]\left(\widetilde{B}_{2}-\lambda \widetilde{B}_{1}\right)^{-1}\left(\widetilde{B}_{2}^{+}-\mu \widetilde{B}_{1}^{+}\right)^{-1}\left[\begin{array}{l}
\widetilde{C}_{1} \\
\widetilde{C}_{2}
\end{array}\right]^{+} J .
\end{aligned}
$$

Proof. The data set

$$
\left(B_{1}\left(B_{2}-\mu B_{1}\right)^{-1}, I_{\mathcal{X}}, C_{1}\left(B_{2}-\mu B_{1}\right)^{-1},\left(C_{2}-\mu C_{1}\right)\left(B_{2}-\mu B_{1}\right)^{-1}, K\right)
$$

satisfies the assumptions (A1)-(A3). Consider the linear relation $A-\mu$

$$
A-\mu=\left\{\left\{\left[\begin{array}{c}
\widetilde{B}_{1}\left(\widetilde{B}_{2}-\mu \widetilde{B}_{1}\right)^{-1} h \\
\widetilde{C}_{1}\left(\widetilde{B}_{2}-\mu \widetilde{B}_{1}\right)^{-1} h
\end{array}\right],\left[\begin{array}{c}
h \\
\left(\widetilde{C}_{2}-\mu \widetilde{C}_{1}\right)\left(\widetilde{B}_{2}-\mu \widetilde{B}_{1}\right)^{-1} h
\end{array}\right]\right\}: h \in \mathcal{H}\right\} .
$$

Due to (4.24) its $\mathcal{L}$-resolvent matrix $W(\lambda)$ satisfies the equality

$$
\begin{aligned}
& {\left[\begin{array}{cc}
-I & 0 \\
\lambda & -I
\end{array}\right]^{-1} W(\lambda)=} \\
& =I_{\mathcal{L} \oplus \mathcal{L}}+i \lambda\left[\begin{array}{c}
\widetilde{C}_{1} \\
\widetilde{C}_{2}-\mu \widetilde{C}_{1}
\end{array}\right]\left(\widetilde{B}_{2}-(\lambda+\mu) \widetilde{B}_{1}\right)^{-1}\left(\widetilde{B}_{2}^{+}-\mu \widetilde{B}_{1}^{+}\right)^{-1}\left[\begin{array}{c}
\widetilde{C}_{1} \\
\widetilde{C}_{2}-\mu \widetilde{C}_{1}
\end{array}\right]^{+} J
\end{aligned}
$$


Then the matrix $W^{\mu}(\lambda)=W(\lambda-\mu)$ is the $\mathcal{L}$-resolvent matrix of $A$ and, hence,

$$
\begin{aligned}
& {\left[\begin{array}{cc}
-I & 0 \\
\lambda & -I
\end{array}\right]^{-1} W^{\mu}(\lambda)=\left[\begin{array}{cc}
-I & 0 \\
\lambda & -I
\end{array}\right]^{-1} W(\lambda-\mu)} \\
& =\left(I_{\mathcal{L} \oplus \mathcal{L}}+i(\lambda-\mu)\left[\begin{array}{c}
\widetilde{C}_{1} \\
\widetilde{C}_{2}
\end{array}\right]\left(\widetilde{B}_{2}-\lambda \widetilde{B}_{1}\right)^{-1}\left(\widetilde{B}_{2}^{+}-\mu \widetilde{B}_{1}^{+}\right)^{-1}\left[\begin{array}{c}
\widetilde{C}_{1} \\
\widetilde{C}_{2}
\end{array}\right]^{+} J\right)\left[\begin{array}{cc}
I & 0 \\
\mu & I
\end{array}\right] .
\end{aligned}
$$

4.3. $\mathcal{L}$-resolvents of $\widehat{A}$. In the case when $\operatorname{ker} P$ is nontrivial we calculated the $\mathcal{L}$ resolvent matrix of the linear relation $A_{0}(\subset \widehat{A})$. A Description of $\mathcal{L}$-resolvents of $A$ is given in Theorem 4.3. In order to obtain a description of $\mathcal{L}$-resolvents of $\widehat{A}$ we will use the same formula and specify the set of parameters $\{p, g\} \in \widetilde{N}(\mathcal{L})$ which correspond to $\mathcal{L}$-resolvents of $\widehat{A}$ via (4.16).

Recall (see [38]) that every generalized resolvent $P_{\mathcal{H}}(\widetilde{A}-\lambda)^{-1} \mid \mathcal{H}$ of $A$ corrresponding to an exit space selfadjoint extension $\widetilde{A}$ in a Hilbert space $\widetilde{\mathcal{H}}=\mathcal{H} \oplus \mathcal{H}_{1}$ can be represented as

$$
P_{\mathcal{H}}(\widetilde{A}-\lambda)^{-1} \mid \mathcal{H}=(T(\lambda)-\lambda)^{-1}, \quad \lambda \in \mathbb{C}_{+},
$$

where $T(\lambda)\left(\lambda \in \mathbb{C}_{+}\right)$is the Strauss family of maximal dissipative linear relations in $\mathcal{H}$ defined by

$$
T(\lambda)=\left\{\left\{P f, P f^{\prime}\right\}:\left\{f, f^{\prime}\right\} \in \widetilde{A}, f^{\prime}-\lambda f \in \mathcal{H}\right\} .
$$

Proposition 4.6. ([15], [17]) Let $\widetilde{A}$ be an exit space selfadjoint extension of $A$, let $T(\lambda)$ be the Strauss family of maximal dissipative linear relations defined by (4.31), let $\left\{\mathcal{L}, \Gamma_{1}, \Gamma_{2}\right\}$ be a boundary triplet for $A^{+}$. Then the pair $\{p, q\} \in \widetilde{N}(\mathcal{L})$ is the Nevanlinna pair corresponding to $\widetilde{A}$ via (4.16) if and only if the pair $\{p, q\}$ is related to $T(\lambda)$ via the formula

$$
\Gamma T(\lambda)=\operatorname{ran}\left[\begin{array}{l}
q(\lambda) \\
p(\lambda)
\end{array}\right]
$$

We will need the following simple statement

Lemma 4.7. Let under the assumptions of Proposition $4.6 \widehat{A}$ be a symmetric extension of $A$. Then:

(i) $\widehat{A} \subset \widetilde{A}$ if and only if $\widehat{A} \subset T(\lambda)$ for some $\lambda \in \mathbb{C}_{+}$;

(ii) $\widehat{A} \subset \widetilde{A}$ if and only if $\Gamma \widehat{A} \subset \operatorname{ran}\left[\begin{array}{l}q(\lambda) \\ p(\lambda)\end{array}\right]$ for some $\lambda \in \mathbb{C}_{+}$.

Proof. 1) The implication $\Rightarrow$ is immediate from (4.31). Conversely, assume that $\widehat{A} \subset T(\lambda)$. In view of (4.31) for every $\left\{g, g^{\prime}\right\} \in \widehat{A}$ there are $\left\{f, f^{\prime}\right\} \in \widetilde{A}$ and $g_{1} \in \mathcal{H}_{1}$ such that

$$
f=g+g_{1}, \quad f^{\prime}=g^{\prime}+\lambda g_{1} .
$$

Hence

$$
\left(f^{\prime}, f\right)_{\widetilde{\mathcal{H}}}=\left(g^{\prime}, g\right)_{\mathcal{H}}+\lambda\left(g_{1}, g_{1}\right)_{\mathcal{H}_{1}} .
$$

Since $\widehat{A}$ and $\widetilde{A}$ are symmetric this implies $g_{1}=0$. Therefore, $\left\{g, g^{\prime}\right\} \in \widetilde{A}$ and hence $\widehat{A} \subset \widetilde{A}$. 
2) The statement (ii) is implied by (i) since the inclusion $\widehat{A} \subset T(\lambda)$ is equivalent to $\Gamma \widehat{A} \subset \Gamma T(\lambda)=\operatorname{ran}\left[\begin{array}{l}q(\lambda) \\ p(\lambda)\end{array}\right]$.

It follows from Lemma 4.7 that all Nevanlinna pairs corresponding to generalized resolvents of $\widehat{A}$ have a common constant part $\Gamma \widehat{A}$.

Lemma 4.8. Let $\widehat{A}$ be the symmetric linear relation (4.3), and let $\left\{\mathcal{L}, \Gamma_{1}, \Gamma_{2}\right\}$ be a boundary triplet for $A^{+}$. Then

(i) $\Gamma \widehat{A}$ is a neutral subspace in $\left(\mathcal{L}^{2}, J_{\mathcal{L}}\right)$ of dimension $\nu:=\operatorname{dim} C \operatorname{ker} K$;

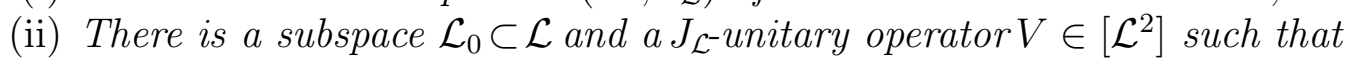

$$
V\left(\{0\} \times \mathcal{L}_{0}\right)=\Gamma \widehat{A} .
$$

Proof. 1) It follows from (4.5) and (4.8) that

$$
\Gamma \widehat{A}=\left\{\left[\begin{array}{l}
\Gamma_{1} \widehat{g} \\
\Gamma_{2} \widehat{g}
\end{array}\right]: \widehat{g} \in \widehat{A}\right\}=\left\{\left[\begin{array}{r}
C_{1} u \\
-C_{2} u
\end{array}\right]: u \in \operatorname{ker} K\right\} .
$$

Clearly, the subspace $\Gamma \widehat{A}$ is finite-dimensional and

$$
\operatorname{dim} \Gamma \widehat{A}=\operatorname{dim} C \operatorname{ker} K \text {. }
$$

The subspace $\Gamma \widehat{A}$ is neutral since for every $u \in \operatorname{ker} K$ one has

$$
\begin{aligned}
\left(\Gamma_{1} \widehat{g}, \Gamma_{2} \widehat{g}\right)-\left(\Gamma_{1} \widehat{g}, \Gamma_{2} \widehat{g}\right) & =\left(C_{1} u, C_{2} u\right)_{\mathcal{L}}-\left(C_{2} u, C_{1} u\right)_{\mathcal{L}} \\
& =K\left(u, B_{1} u\right)-K\left(B_{1} u, u\right)=0 .
\end{aligned}
$$

2) Let us decompose $\mathcal{L}$ into the orthogonal sum

$$
\mathcal{L}=\mathcal{L}_{0} \oplus \mathcal{L}_{1}
$$

where $\mathcal{L}_{0}$ is a subspace of $\mathcal{L}$ such that $\operatorname{dim} \mathcal{L}_{0}=\nu$. The subspace $\{0\} \times \mathcal{L}_{0}$ is $J_{\mathcal{L}^{-}}$ neutral and hence there exists a $J_{\mathcal{L}}$-unitary operator $V \in\left[\mathcal{L}^{2}\right]$ such that $V\left(\{0\} \times \mathcal{L}_{0}\right)=$ $\Gamma \widehat{A}$.

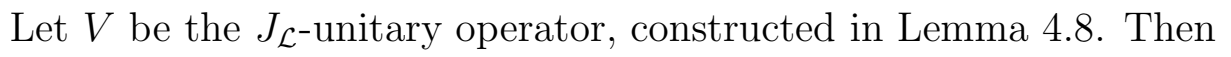

$$
\widehat{W}(\lambda)=\left(\widehat{w}_{i j}(\lambda)\right)_{i, j=1}^{2}:=W_{\Pi \mathcal{L}}(\lambda) V
$$

is also the $\mathcal{L}$-resolvent matrix of $A_{0}$ with the advantage that the $\mathcal{L}$-resolvents of $\widehat{A}$ can be easily described in its terms.

Proposition 4.9. The set of all $\mathcal{L}$-resolvents of $\widehat{A}$ is parametrized by the formula

$$
\left.P_{\mathcal{L}}(\widetilde{A}-\lambda)^{-1}\right|_{\mathcal{L}}=\left(\widehat{w}_{11}(\lambda) q(\lambda)+\widehat{w}_{12}(\lambda) p(\lambda)\right)\left(\widehat{w}_{21}(\lambda) q(\lambda)+\widehat{w}_{22}(\lambda) p(\lambda)\right)^{-1}
$$

where $\{p, q\}$ ranges over the set $\widetilde{\mathcal{N}}(\mathcal{L})$ of Nevanlinna pairs of the form

$$
p(\lambda)=\left[\begin{array}{cc}
I_{\mathcal{L}_{0}} & 0 \\
0 & p_{1}(\lambda)
\end{array}\right], \quad q(\lambda)=\left[\begin{array}{cc}
0_{\mathcal{L}_{0}} & 0 \\
0 & q_{1}(\lambda)
\end{array}\right], \quad\left\{p_{1}, q_{1}\right\} \in \tilde{N}\left(\mathcal{L}_{1}\right) .
$$

Proof. Let $\{\widetilde{p}, \widetilde{q}\}$ be a Nevanlinna pair defined by

$$
\left[\begin{array}{l}
\widetilde{q}(\lambda) \\
\widetilde{p}(\lambda)
\end{array}\right]=V\left[\begin{array}{l}
q(\lambda) \\
p(\lambda)
\end{array}\right], \quad\{p, q\} \in \widetilde{\mathcal{N}}(\mathcal{L})
$$


It follows from Lemma 4.7 that the formula

$$
\left.P_{\mathcal{L}}(\widetilde{A}-\lambda)^{-1}\right|_{\mathcal{L}}=\left(w_{11}(\lambda) \widetilde{q}(\lambda)+w_{12}(\lambda) \widetilde{p}(\lambda)\right)\left(w_{21}(\lambda) \widetilde{q}(\lambda)+w_{22}(\lambda) \widetilde{p}(\lambda)\right)^{-1}
$$

establishes a 1-1 correspondence between the set of all $\mathcal{L}$-resolvents of $\widehat{A}$ and the set of Nevanlinna families $\{\widetilde{p}, \widetilde{q}\}$ such that

$$
\Gamma \widehat{A} \subset \operatorname{ran}\left[\begin{array}{l}
\widetilde{q}(\lambda) \\
\widetilde{p}(\lambda)
\end{array}\right], \quad \lambda \in \mathbb{C}_{+} .
$$

Since

$$
\Gamma \widehat{A}=V\left[\begin{array}{c}
0 \\
\mathcal{L}_{0}
\end{array}\right], \quad \text { and } \operatorname{ran}\left[\begin{array}{l}
\widetilde{q}(\lambda) \\
\widetilde{p}(\lambda)
\end{array}\right]=V \operatorname{ran}\left[\begin{array}{c}
q(\lambda) \\
p(\lambda)
\end{array}\right],
$$

the inclusion (4.36) is equivalent to the inclusion

$$
\left[\begin{array}{c}
0 \\
\mathcal{L}_{0}
\end{array}\right] \subset \operatorname{ran}\left[\begin{array}{c}
q(\lambda) \\
p(\lambda)
\end{array}\right], \quad \lambda \in \mathbb{C}_{+},
$$

which, in turn, means that the pair $\{p, q\}$ admits the representation (4.35).

4.4. Description of AIP solutions. To describe solutions of the AIP it remains to combine Theorem 3.3 and Proposition 4.9, Let the ovf $\Theta(\lambda)$ be defined by

$$
\Theta(\lambda)=\left[\begin{array}{ll}
I & 0 \\
\lambda & I
\end{array}\right] \widehat{W}(\lambda)=\left(I_{\mathcal{L} \oplus \mathcal{L}}-\lambda\left[\begin{array}{l}
\widetilde{C}_{1} \\
\widetilde{C}_{2}
\end{array}\right]\left(I_{\mathcal{H}}-\lambda \widetilde{B}_{1}\right)^{-1}\left[\widetilde{C}_{2}^{+}-\widetilde{C}_{1}^{+}\right]\right) V
$$

Theorem 4.10. Let the AIP data set satisfy $(A 1)-(A 3)$. Then the formula

$$
\left[\begin{array}{l}
\psi(\lambda) \\
\phi(\lambda)
\end{array}\right]=\Theta(\lambda)\left[\begin{array}{l}
q(\lambda) \\
p(\lambda)
\end{array}\right]\left(\widehat{w}_{21}(\lambda) q(\lambda)+\widehat{w}_{22}(\lambda) p(\lambda)\right)^{-1}
$$

establishes the 1-1 correspondence between the set of all normalized solutions $\{\phi, \psi\}$ of $\operatorname{AIP}\left(B_{1}, B_{2}, C_{1}, C_{2}, K\right)$ and the set of all equivalence classes of Nevanlinna pairs $\{p, q\} \in \widetilde{N}(\mathcal{L})$ of the form (4.35). The corresponding mapping $F: \mathcal{X} \rightarrow \mathcal{H}(\phi, \psi)$ in (C1), (C2) is uniquely defined by the solution $\{\phi, \psi\}$ :

$$
(F h)(\lambda)=[\varphi(\lambda)-\psi(\lambda)] \widetilde{G}(\lambda) \mathcal{P}_{\mathcal{X}_{0}} h \quad\left(\lambda \in \mathcal{O}_{ \pm}\right),
$$

where

$$
\widetilde{G}(\lambda)=\left[\begin{array}{c}
\widetilde{C}_{1} \\
\widetilde{C}_{2}
\end{array}\right]\left(I_{\mathcal{H}}-\left.\lambda \widetilde{B}_{1}\right|_{\mathcal{X}_{0}}\right)^{-1} \quad\left(\lambda \in \mathcal{O}_{ \pm}\right),
$$

and $\mathcal{P}_{\mathcal{X}_{0}}$ is the skew projection onto $\mathcal{X}_{0}$ in the decomposition (4.1).

Proof. Indeed the description (4.39) is implied by (3.3), (4.34) and (4.38).

Next, let $g \in\left(B_{2}-\mu B_{1}\right) \mathcal{X}_{0}\left(\mu \in \mathcal{O}_{ \pm}\right)$. Applying $(\mathrm{C} 1)$ to the vector

$$
h=h_{\mu}:=\left(B_{2}-\mu B_{1}\right)^{-1} g,
$$

one obtains

$$
\begin{aligned}
(F g)(\lambda) & =\left(F B_{2} h_{\mu}\right)(\lambda)-\mu\left(F B_{1} h_{\mu}\right)(\lambda) \\
& =[\varphi(\lambda)-\psi(\lambda)] G(\mu) g+(\lambda-\mu)\left(F B_{1} h_{\mu}\right)(\lambda) .
\end{aligned}
$$

Setting in (4.41) $\lambda=\mu$, one obtains

$$
(F g)(\mu))=[\varphi(\mu)-\psi(\mu)] \widetilde{G}(\mu) g \quad\left(\mu \in \mathcal{O}_{ \pm}, g \in \mathcal{X}\right) .
$$

The equality (4.42) holds for every $g \in\left(B_{2}-\mu B_{1}\right) \mathcal{X}_{0}\left(\mu \in \mathcal{O}_{ \pm}\right)$. 
Let $g \in \mathcal{X}_{0}$ and let $g_{n} \in\left(B_{2}-\mu B_{1}\right) \mathcal{X}_{0}$ and $g_{n} \rightarrow g$. Then taking the limit in

$$
\left(F g_{n}\right)(\mu)=[\varphi(\mu)-\psi(\mu)] \widetilde{G}(\mu) g_{n}
$$

one obtains (4.40) for $g \in \mathcal{X}_{0}$. To prove (4.40) it remains to notice that $F g \equiv 0$ for all $g \in \operatorname{ker} K$.

Theorem 4.11. Let the AIP data set satisfy $(A 1),(A 2),\left(A 3^{\prime}\right)$ and let

$$
\begin{aligned}
\Theta^{\mu}(\lambda) & =\left[\begin{array}{ll}
I & 0 \\
\lambda & I
\end{array}\right] \widehat{W}^{\mu}(\lambda) \\
& =\left(I+i(\lambda-\mu)\left[\begin{array}{c}
\widetilde{C}_{1} \\
\widetilde{C}_{2}
\end{array}\right]\left(\widetilde{B}_{2}-\lambda \widetilde{B}_{1}\right)^{-1}\left(\widetilde{B}_{2}^{+}-\mu \widetilde{B}_{1}^{+}\right)^{-1}\left[\begin{array}{l}
\widetilde{C}_{1} \\
\widetilde{C}_{2}
\end{array}\right]^{+} J\right) V .
\end{aligned}
$$

Then the formula

$$
\left[\begin{array}{l}
\psi(\lambda) \\
\phi(\lambda)
\end{array}\right]=\Theta^{\mu}(\lambda)\left[\begin{array}{l}
q(\lambda) \\
p(\lambda)
\end{array}\right]\left(\widehat{w}_{21}^{\mu}(\lambda) q(\lambda)+\widehat{w}_{22}^{\mu}(\lambda) p(\lambda)\right)^{-1}
$$

establishes the 1-1 correspondence between the set of all normalized solutions $\{\phi, \psi\}$ of $\operatorname{AIP}\left(B_{1}, B_{2}, C_{1}, C_{2}, K\right)$ and the set of all equivalence classes of Nevanlinna pairs $\{p, q\} \in \widetilde{N}(\mathcal{L})$ of the form (4.35). The corresponding mapping $F: \mathcal{X} \rightarrow \mathcal{H}(\phi, \psi)$ in (C1), (C2) is uniquely defined by the formula (4.40).

Corollary 4.12. Let the AIP data set satisfies $(A 1)-(A 3)$ and let $\operatorname{ran} C_{2}=\mathcal{L}$. Then the formula

$$
m(\lambda)=\left(\theta_{11}(\lambda) q(\lambda)+\theta_{12}(\lambda) p(\lambda)\right)\left(\theta_{21}(\lambda) q(\lambda)+\theta_{22}(\lambda) p(\lambda)\right)^{-1}
$$

establishes the 1-1 correspondence between the set of all solutions $m(\lambda)$ of $\operatorname{AIP}\left(B_{1}, B_{2}, C_{1}, C_{2}, K\right)$ and the set of all equivalence classes of Nevanlinna pairs $\{p, q\} \in \widetilde{N}(\mathcal{L})$ of the form (4.35).

In the case when the AIP data set satisfy $(A 1),(A 2)$, and $\left(A 3^{\prime}\right)$ similar formula can be written in terms of the mvf $\Theta^{\mu}(\lambda)$.

\section{EXAmples}

5.1. Tangential interpolation problem. Let $\lambda_{j} \in \mathbb{C}_{+}, \xi_{j} \in \mathbb{C}^{d}, \eta_{j} \in \mathbb{C}^{d}(1 \leq$ $j \leq n)$. Consider the following problem. Find $m \in N^{d \times d}:=N\left(\mathbb{C}^{d}\right)$ such that

$$
m\left(\lambda_{j}\right) \eta_{j}=\xi_{j} \quad(1 \leq j \leq n) .
$$

The problem (5.1) is called tangential (or one-sided) interpolation problem and was considered first by I. Fedchina [23] in the Schur class. In [25], 28] the inclusion of this (and more general bitangential) problem into the scheme of AIP was demonstrated.

For the case of Nevanlinna class let us set $B_{1}=I_{n}$,

$$
B_{2}=\operatorname{diag}\left(\lambda_{1} \ldots \lambda_{n}\right), \quad C_{1}=\left[\begin{array}{lll}
\xi_{1} & \cdots & \xi_{n}
\end{array}\right], \quad C_{2}=\left[\begin{array}{lll}
\eta_{1} & \cdots & \eta_{n}
\end{array}\right],
$$

and let

$$
P=\left[\frac{\eta_{k}^{*} \xi_{j}-\xi_{k}^{*} \eta_{j}}{\lambda_{j}-\bar{\lambda}_{k}}\right]_{j, k=1}^{n}
$$

be the unique solution of the Lyapunov equation

$$
P B_{2}-B_{2}^{*} P=C_{2}^{*} C_{1}-C_{1}^{*} C_{2} .
$$


Assume that $P$ is nonnegative and nondegenerate and that $\operatorname{ran} C_{2}=\mathbb{C}^{d}$. Then the data set $\left(B_{1}, B_{2}, C_{1}, C_{2}, P\right)$ satisfies the assumptions $(A 1)-(A 3)$. Consider the AIP corresponding to this data set. Due to Lemma 2.9 and Proposition 3.5 every AIP solution is equivalent to a mvf $m(\cdot) \in N^{d \times d}$ and the mapping $F: \mathcal{H} \rightarrow \mathcal{H}(m)$ in $(C 1),(C 2)$ is uniquely defined by (4.40). Therefore, the conditions $(C 1),(C 2)$ can be rewritten as

$$
\begin{gathered}
(F u)(\lambda):=\left[\begin{array}{ll}
I_{d} & -m(\lambda)
\end{array}\right]\left[\begin{array}{l}
C_{1} \\
C_{2}
\end{array}\right]\left(B_{2}-\lambda\right)^{-1} u \in \mathcal{H}(m) \quad\left(u \in \mathbb{C}^{n}\right) \\
\|F u\|_{\mathcal{H}(m)}^{2} \leq(P u, u) \quad\left(u \in \mathbb{C}^{n}\right) .
\end{gathered}
$$

We claim that the problem (5.1) is equivalent to the problem (5.4), (5.5). Indeed, the condition $(C 1)$ takes the form

$$
\left[\frac{\xi_{j}-m(\lambda) \eta_{j}}{\lambda-\lambda_{j}}\right]_{j=1}^{n} u \in \mathcal{H}(m), \quad u \in \mathbb{C}^{n},
$$

which is equivalent to $(B 1)$. Moreover, if $m(\cdot)$ has the integral representation

$$
m(\lambda)=\alpha+\beta \lambda+\int_{\mathbb{R}}\left(\frac{1}{t-\lambda}-\frac{t}{1+t^{2}}\right) d \sigma(t),
$$

where $\alpha, \beta \in \mathbb{C}^{d \times d}, \alpha=\alpha^{*}, \beta \geq 0$ and $\sigma(t)$ is a nondecreasing $d \times d$-valued mvf, then the vvf $(F u)(\lambda)$ takes the form

$$
(F u)(\lambda)=\left[\beta \eta_{j}+\int_{\mathbb{R}} \frac{d \sigma(t) \eta_{j}}{(t-\lambda)\left(t-\lambda_{j}\right)}\right]_{j=1}^{n} u .
$$

Due to [4, Theorem 2.5] one obtains

$$
\begin{aligned}
\|F u\|_{\mathcal{H}(m)}^{2} & =u^{*}\left[\eta_{k}^{*}\left(\beta+\int_{\mathbb{R}} \frac{d \sigma(t)}{\left(t-\lambda_{j}\right)\left(t-\bar{\lambda}_{k}\right)}\right) \eta_{j}\right]_{j, k=1}^{n} u \\
& =u^{*}\left[\eta_{k}^{*} \frac{m\left(\lambda_{j}\right)-m\left(\lambda_{k}\right)^{*}}{\lambda_{j}-\bar{\lambda}_{k}} \eta_{j}\right]_{j, k=1}^{n} u
\end{aligned}
$$

Thus, (5.5) is implied by (5.6) and (5.1).

More general bitangential interpolation problems in the classes of Nevanlinna pairs with multiple points can be included in the AIP by using the data set $\left(B_{1}, B_{2}, C_{1}, C_{2}, P\right)$ :

(1) $B_{1}=I_{N}, B_{2}=\operatorname{diag}\left(J\left(\lambda_{1}\right) \ldots J\left(\lambda_{\ell}\right)\right), \lambda_{j} \in \mathbb{C} \backslash \mathbb{R}(1 \leq j \leq \ell)$, and $J\left(\lambda_{j}\right)$ is a Jordan cell, corresponding to the eigenvalue $\lambda_{j}$

$$
J\left(\lambda_{j}\right)=\left[\begin{array}{cccc}
\lambda_{j} & 1 & & \\
& \ddots & \ddots & \\
& & \ddots & 1 \\
& & & \lambda_{j}
\end{array}\right], \quad(1 \leq j \leq \ell)
$$

of the order $n_{j}, n=n_{1}+n_{2}+\cdots+n_{\ell}$.

(2) $C_{1}=\left[\begin{array}{lll}\xi_{1} & \cdots & \xi_{n}\end{array}\right], \quad C_{2}=\left[\begin{array}{lll}\eta_{1} & \cdots & \eta_{n}\end{array}\right]$;

(3) $P$ is a nonnegative solution $P$ of (5.3). 
If the set $\left\{\lambda_{j}\right\}_{j=1}^{\ell}$ contains symmetric points then the solution $P$ of the Lyapunov equation (5.3) is not unique. Assume that there is a nonnegative nondegenerate solution $P$ of (5.3) . Then the AIP corresponding to the data set $\left(B_{1}, B_{2}, C_{1}, C_{2}, P\right)$ can be formulated as follows. Find a Nevanlinna pair $\{\varphi, \psi\}$ such that:

$$
\begin{gathered}
(F u)(\lambda):=[\varphi(\lambda)-\psi(\lambda)]\left[\begin{array}{l}
C_{1} \\
C_{2}
\end{array}\right]\left(B_{2}-\lambda\right)^{-1} u \in \mathcal{H}(m) \quad\left(u \in \mathbb{C}^{n}\right) ; \\
\|F u\|_{\mathcal{H}(m)}^{2} \leq(P u, u) \quad\left(u \in \mathbb{C}^{n}\right) .
\end{gathered}
$$

One can show that every solution $\{\varphi, \psi\}$ of the problem (5.7), (5.8) satisfies the Parseval equality

$$
\|F u\|_{\mathcal{H}(m)}^{2}=(P u, u) \quad\left(u \in \mathbb{C}^{n}\right) .
$$

Regular bitangential interpolation problems in the Schur and Nevanlinna classes were studied in [36], [9], 20], 28], 8], 6], 3]. Singular tangential and bitangential interpolation problems considered in [24], [36], [20], [14], [12], [21] can be included in the above consideration by imposing the assumption (A2) on the data set.

5.2. Hamburger moment problem. Let $s_{j} \in \mathbb{C}^{d \times d}, j \in \mathbb{N}$ and let $S_{n}$ be the Hankel block matrix

$$
S_{n}=\left(s_{i+j}\right)_{i, j=0}^{n} .
$$

A $\mathbb{C}^{d \times d}$ - valued nondecreasing right continuous function $\sigma(t)$ is called a solution of the Hamburger moment problem if

$$
\int t^{j} d \sigma(t)=s_{j} \quad(j \in \mathbb{N}) .
$$

It is known that the Hamburger moment problem (5.9) is solvable iff $S_{n} \geq 0$ for all $n \in \mathbb{N}$. Due to Hamburger-Nevanlinna theorem a function $\sigma(t)$ is a solution of the Hamburger moment problem (5.9) if and only if the associated mvf

$$
m(\lambda)=\int_{-\infty}^{\infty} \frac{d \sigma(t)}{t-\lambda}
$$

has the following nontangential asymptotic at $\infty$

$$
m(\lambda) \sim-\frac{s_{0}}{\lambda}-\frac{s_{1}}{\lambda^{2}}-\cdots-\frac{s_{2 n}}{\lambda^{2 n+1}}+O\left(\frac{1}{\lambda^{2 n+2}}\right) \quad(\lambda \hat{\rightarrow} \infty)
$$

for every $n \in \mathbb{N}$.

Let $\mathcal{L}=\mathbb{C}^{d}$, let $\mathcal{X}$ be the space of all vector polynomials

$$
h(X)=\sum_{j=0}^{n} h_{j} X^{j}, \quad h_{j} \in \mathcal{L},
$$

and let the nonnegative form $K(h, h)$ be defined by

$$
K(h, h)=\sum_{j, k=0}^{n}\left(s_{j+k} h_{j}, h_{k}\right)_{\mathcal{L}} .
$$

Assume that all the matrices are nondegenerate and consider the closure $\mathcal{H}$ of the space $\mathcal{X}$ endowed with the inner product $K(\cdot, \cdot)$. Then the closure $M$ of the multiplication operator $M_{0}$ in $\mathcal{X}$ is a symmetric operator in $\mathcal{H}$. The moment problem (5.9) is called indeterminate if the defect numbers of $M$ are equal to $d$. As was shown 
in [10] the scalar moment problem $(d=1)$ is indeterminate if and only if there exists $\delta>0$ such that

$$
S_{n} \geq \delta>0 \quad \text { for all } n \in \mathbb{N} \text {. }
$$

Slight modification of the proof of this statement in [10] shows that the condition (5.13) is also necessary and sufficient for the moment problem (5.9) to be indeterminate for arbitrary $d$.

Let us consider the abstract interpolation problem in the class $N^{d \times d}$ corresponding to the indeterminate moment problem (5.9). Define the operators $B_{1}, B_{2}: \mathcal{X} \rightarrow \mathcal{X}$ and $C_{1}, C_{2}: \mathcal{X} \rightarrow \mathcal{L}$ by the equalities

$$
B_{1} h=\frac{h(X)-h_{0}}{X}, \quad B_{2} h=h, \quad C_{1} h=\sum_{j=1}^{n} s_{j-1} h_{j}, \quad C_{2} h=-h(0) .
$$

Then the data set $\left(B_{1}, B_{2}, C_{1}, C_{2}, K\right)$ satisfies the assumption (A1). (A2) is clearly in force, since ker $K=\{0\}$. Let us show that (A3) is also in force.

Proposition 5.1. The operators $B_{1}, C_{1}, C_{2}$ admit continuous extensions to the operators $\widetilde{B}_{1} \in[\mathcal{H}]$, and $\widetilde{C}_{1}, \widetilde{C}_{2} \in[\mathcal{H}, \mathcal{L}]$.

Proof. Let $\widetilde{B}_{1}$ be the closure of the graph of the operator $B_{1}$. Then

$$
\widetilde{B}_{1}^{-1}=\{\{h, M h+u\}: h \in \operatorname{dom} M, u \in \mathcal{L}\} .
$$

As was shown in 31] $\rho(M, \mathcal{L})=\mathbb{C}$ in the case of indeterminate moment problem (5.9). In particular, $0 \in \rho(M, \mathcal{L})$, that is

$$
\operatorname{ran} \widetilde{B}_{1}^{-1}=\operatorname{ran} M \dot{+} \mathcal{L}=\mathcal{H}, \quad \operatorname{ker} \widetilde{B}_{1}^{-1}=\operatorname{ran} M \cap \mathcal{L}=\{0\} .
$$

Therefore $\widetilde{B}_{1}$ is the graph of a bounded operator in $\mathcal{H}$ for which we will keep the same notation.

It follows from (5.13) that for every polynomial $h \in \mathcal{X}$

$$
\begin{aligned}
\|h\|_{\mathcal{H}}^{2} & =K(h, h)=\sum_{j, k=0}^{n} h_{k}^{*} s_{j+k} h_{j} \\
& \geq \delta \sum_{j, k=0}^{n}\left\|h_{j}\right\|^{2} \geq \delta\left\|h_{0}\right\|^{2} .
\end{aligned}
$$

Therefore,

$$
\left\|C_{2} h\right\|^{2} \leq \frac{1}{\delta}\|h\|_{\mathcal{H}}^{2}
$$

and, hence, the operator $C_{2}: \mathcal{X} \subset \mathcal{H} \rightarrow \mathcal{L}$ is bounded. Let us note that the boundedness of $C_{2}$ is implied also by the fact that $0 \in \rho(M, \mathcal{L})$, since $C_{2} h=\mathcal{P}_{M, \mathcal{L}}(0) h$, where $\mathcal{P}_{M, \mathcal{L}}(\lambda)$ is the skew projection onto $\mathcal{L}$ in the decomposition $\mathcal{H} \oplus \mathcal{L}=\operatorname{ran}(M-\lambda) \dot{+} \mathcal{L}$.

The boundedness of $C_{1}: \mathcal{X} \subset \mathcal{H} \rightarrow \mathcal{L}$ is implied by the equality

$$
\left(C_{1} h, u\right)_{\mathcal{L}}=K\left(\frac{h(X)-h(0)}{X}, u\right)=K\left(B_{1} h, u\right)
$$


Indeed, it follows from (5.15) that

$$
\begin{aligned}
\left|\left(C_{1} h, u\right)_{\mathcal{L}}\right| & \leq K\left(B_{1} h, B_{1} h\right)^{1 / 2} K(u, u)^{1 / 2} \\
& =\left\|B_{1} h\right\|_{\mathcal{H}}\left(s_{0} u, u\right)^{1 / 2} \\
& \leq\left\|B_{1}\right\|\left\|s_{0}^{1 / 2}\right\|\|h\|_{\mathcal{H}}\|u\|_{\mathcal{L}}
\end{aligned}
$$

and hence $C_{1}$ is bounded and

$$
\left\|C_{1}\right\| \leq\left\|B_{1}\right\|\left\|s_{0}^{1 / 2}\right\|
$$

Remark 5.2. The definition (5.14) of $C_{1}$ can be rewritten as

$$
C_{1} h=\widetilde{h}(0),
$$

where the adjacent polynomial $\widetilde{h}$ is defined by

$$
(\widetilde{h}(\lambda), u)_{\mathcal{L}}=K\left(\frac{h(X)-h(\lambda)}{X-\lambda}, u\right), \quad u \in \mathcal{L} .
$$

Let us consider the $\left[\mathcal{H}, \mathcal{L}^{2}\right]$-valued operator function

$$
G(\lambda)=\left[\begin{array}{l}
C_{1} \\
C_{2}
\end{array}\right]\left(I-\lambda B_{1}\right)^{-1}, \quad \lambda \in \mathbb{C} .
$$

Recall some useful formulas (see [29])

$$
\begin{gathered}
\left(I-\lambda B_{1}\right)^{-1} h=\frac{X h(X)-\lambda h(\lambda)}{X-\lambda}, \quad(h \in \mathcal{X}) . \\
C_{1}\left(I-\lambda B_{1}\right)^{-1} h=\widetilde{h}(\lambda), \quad C_{2}\left(I_{m}-\lambda B_{1}\right)^{-1} h=-h(\lambda) .
\end{gathered}
$$

The corresponding abstract interpolation problem can be formulated as follows. Find a Nevanlinna pair $\{\varphi, \psi\} \in \widetilde{N}\left(\mathbb{C}^{d}\right)$, such that

$$
\begin{gathered}
F h:=[\varphi(\lambda)-\psi(\lambda)] G(\lambda) h \in \mathcal{H}(\varphi, \psi) ; \\
\|F h\|_{\mathcal{H}(\varphi, \psi)}^{2} \leq K(h, h)
\end{gathered}
$$

for all $h \in \mathcal{X}$.

Since the operators $B_{1}, B_{2}=I$ satisfy the assumption (U) the mapping $F: \mathcal{X} \rightarrow$ $\mathcal{H}(\varphi, \psi)$ corresponding to the solution $\{\varphi, \psi\}$ of the AIP is uniquely defined (see Proposition 4.40). Moreover, since $\operatorname{ran} C_{2}=\mathcal{L}$ it follows from Lemma 2.9 that any solution $\{\varphi, \psi\}$ of the AIP is equivalent to a pair $\left\{I_{d}, m(\lambda)\right\}$, where $m \in N^{d \times d}$.

Theorem 5.3. Let $m$ be a solution of the $A I P\left(B_{1}, B_{2}, C_{1}, C_{2}, K\right)$, which assumes the integral representation (5.29), and let $F: \mathcal{X} \rightarrow \mathcal{H}(\varphi, \psi)$ be the mapping corresponding to the solution $m$ and given by the formula (5.21). Then:

(1) $\sigma$ is a solution of the Hamburger moment problem (5.9);

(2) for every polynomial $h \in \mathcal{X}$ one has

$$
\begin{gathered}
(F h)(\lambda)=\int_{-\infty}^{\infty} \frac{d \sigma(t) h(t)}{t-\lambda}, \\
\|F h\|_{\mathcal{H}(m)}^{2}=K(h, h) .
\end{gathered}
$$


Proof. For a monic polynomial $h=u X^{j}(u \in \mathcal{L})$ one obtains from (5.14)-(5.18)

$$
\begin{aligned}
(G h)(\lambda) & =\left[\begin{array}{l}
C_{1} \\
C_{2}
\end{array}\right]\left(X^{j}+\lambda X^{j-1}+\cdots+\lambda^{j}\right) u \\
& =\left[\begin{array}{c}
\left(\lambda^{j-1} s_{0}+\cdots+\lambda s_{j-2}+s_{j-1}\right) u \\
-\lambda^{j} u
\end{array}\right]
\end{aligned}
$$

and hence

$$
\begin{aligned}
(F h)(\lambda) & =\left[\begin{array}{ll}
I_{d} & -m(\lambda)
\end{array}\right] G(\lambda) h \\
& =\left(\lambda^{j} m(\lambda)+\lambda^{j-1} s_{0}+\cdots+s_{j-1}\right) u \\
& =\int_{-\infty}^{\infty} \frac{t^{j}}{t-\lambda} d \sigma(t) u .
\end{aligned}
$$

When $j=0$ it follows from (5.25) and Lemma 2.10 that $m(\lambda)=O(1)$ if $\lambda \widehat{\rightarrow} \infty$. Setting $j=1$ one derives from (5.25) that

$$
\lambda m(\lambda)+s_{0}=O(1) \quad(\lambda \widehat{\rightarrow} \infty) .
$$

Therefore, $m(\lambda)=O\left(\frac{1}{\lambda}\right)$ and applying Lemma 2.10, (ii) gives

$$
m(\lambda)+\frac{s_{0}}{\lambda}=O\left(\frac{1}{\lambda^{2}}\right) .
$$

And similarly, for $h=u X^{n}$ one obtains from $(\mathrm{C} 1)\left(\lambda^{n} m(\lambda)+s_{0} \lambda^{n-1}+\cdots+s_{n-1}\right) u \in$ $\mathcal{H}(m)$, or by Lemma 2.10, (ii)

$$
m(\lambda)+\frac{s_{0}}{\lambda}+\cdots+\frac{s_{n-1}}{\lambda^{n}}=O\left(\frac{1}{\lambda^{n+1}}\right)
$$

for arbitrary $n \in \mathbb{N}$. In view of Hamburger-Nevanlinna theorem this implies that $\sigma$ is a solution of the Hamburger moment problem (5.9).

For arbitrary polynomial $h=\sum_{j=0}^{n} u_{j} X^{j} \in \mathcal{X}$ the formula (5.25) can be rewritten as

$$
(F h)(\lambda)=\int_{-\infty}^{\infty} \frac{d \sigma(t) h(t)}{t-\lambda} .
$$

Applying the formula for the inner product in $\mathcal{H}(m)$ (see [4, Theorem 2.5]) one obtains

$$
\|F h\|_{\mathcal{H}(m)}^{2}=\int_{-\infty}^{\infty}(d \sigma(t) h(t), h(t))=\sum_{j, k=0}^{n}\left(s_{j+k} u_{j}, u_{k}\right)_{\mathcal{L}}=K(h, h) .
$$

This proves (5.24).

Conversely, let $\sigma$ be a solution of the Hamburger moment problem (5.9). Then it follows from (5.23) and Theorem 2.5 from [4] that $F h \in \mathcal{H}(m)$ for arbitrary polynomial $h \in \mathcal{X}$. This proves $(\mathrm{C} 1)$. (C2) is implied by the equality (5.24).

To calculate the $\mathcal{L}$-resolvent matrix let us introduce a system of matrix polynomials $\left\{P_{n}(\lambda)\right\}_{n=0}^{\infty}$ orthogonal with respect to the form $K$ :

$$
K\left(P_{j} u, P_{k} v\right)=v^{*} u \delta_{j k}, \quad j, k \in \mathbb{N}
$$

and a system of adjacent polynomials $\left\{\widetilde{P}_{k}(\lambda)\right\}_{n=0}^{\infty}$

$$
v^{*} \widetilde{P}_{k}(\lambda) u=K\left(\frac{P_{k}(t)-P_{k}(\lambda)}{t-\lambda} u, v\right) \quad k \in \mathbb{N} .
$$


Proposition 5.4. For every $u \in \mathcal{L}$ the following formulas hold

$$
C_{1}^{*} u=\sum_{k=1}^{\infty} P_{k}(t) \widetilde{P}_{k}(0)^{*} u, \quad C_{2}^{*} u=-\sum_{k=0}^{\infty} P_{k}(t) P_{k}(0)^{*} u .
$$

Proof. Indeed, for every $j \in \mathbb{N} \cup\{0\}, u, v \in \mathcal{L}$ one obtains from (5.14), (5.16)

$$
\begin{aligned}
\left(C_{1}^{*} u, P_{j} v\right)_{\mathcal{H}} & =\left(u, C_{1} P_{j} v\right)_{\mathcal{L}}=\left(u, \widetilde{P}_{j}(0) v\right)_{\mathcal{L}} \\
& =\left(\widetilde{P}_{j}(0)^{*} u, v\right)_{\mathcal{H}}=\left(\sum_{k=1}^{\infty} P_{k}(t) \widetilde{P}_{k}(0)^{*} u, P_{j} v\right)_{\mathcal{H}}, \\
\left(C_{2}^{*} u, P_{j} v\right)_{\mathcal{H}} & =\left(u, C_{2} P_{j} v\right)_{\mathcal{L}}=-\left(u, P_{j}(0) v\right)_{\mathcal{L}} \\
& =-\left(P_{j}(0)^{*} u, v\right)_{\mathcal{H}}=-\left(\sum_{k=1}^{\infty} P_{k}(t) P_{k}(0)^{*} u, P_{j} v\right)_{\mathcal{H}},
\end{aligned}
$$

Applying the formulas (4.22), (5.20) and (5.26) one obtains the resolvent matrix $\Theta(\lambda)$ :

$$
\begin{aligned}
& \theta_{11}(\lambda) u=u-\lambda \widetilde{C}_{1}\left(I-\lambda \widetilde{B}_{1}\right)^{-1}\left(\sum_{k=0}^{\infty} P_{k}(t) P_{k}(0)^{*} u\right)=\left(I+\lambda \sum_{k=0}^{\infty} \widetilde{P}_{k}(\lambda) P_{k}(0)^{*}\right) u \\
& \theta_{12}(\lambda) u=\lambda \widetilde{C}_{1}\left(I-\lambda \widetilde{B}_{1}\right)^{-1}\left(\sum_{k=0}^{\infty} P_{k}(t) \widetilde{P}_{k}(0)^{*} u\right)=\lambda \sum_{k=0}^{\infty} \widetilde{P}_{k}(\lambda) \widetilde{P}_{k}(0)^{*} u \\
& \theta_{21}(\lambda) u=-\lambda \widetilde{C}_{2}\left(I-\lambda \widetilde{B}_{1}\right)^{-1}\left(\sum_{k=0}^{\infty} P_{k}(t) P_{k}(0)^{*} u\right)=-\lambda \sum_{k=0}^{\infty} P_{k}(\lambda) P_{k}(0)^{*} u \\
& \theta_{22}(\lambda) u=u+\lambda \widetilde{C}_{2}\left(I-\lambda \widetilde{B}_{1}\right)^{-1}\left(\sum_{k=1}^{\infty} P_{k}(t) \widetilde{P}_{k}(0)^{*} u\right)=\left(I-\lambda \sum_{k=1}^{\infty} P_{k}(\lambda) \widetilde{P}_{k}(0)^{*}\right) u .
\end{aligned}
$$

Application of general result in Corollary 4.12 gives the well known description of solutions of the moment problem (5.9)

$$
\int_{\mathbb{R}} \frac{d \sigma(t)}{t-\lambda}=\left(\theta_{11}(\lambda) q(\lambda)+\theta_{12}(\lambda) p(\lambda)\right)\left(\theta_{21}(\lambda) q(\lambda)+\theta_{22}(\lambda) p(\lambda)\right)^{-1}
$$

when the pair $\{p, q\}$ ranges over the class $N\left(\mathbb{C}^{d}\right)$.

5.3. Truncated Hamburger moment problem. Let $s_{0}, s_{1}, \ldots, s_{2 n} \in \mathbb{C}^{d \times d}$. A $\mathbb{C}^{d \times d}$ - valued nondecreasing right continuous function $\sigma(\lambda)$ is called a solution of the truncated Hamburger moment problem if

$$
\begin{gathered}
\int t^{j} d \sigma(t)=s_{j} \quad(j=0,1, \ldots, 2 n-1) \\
\int t^{2 n} d \sigma(t) \leq s_{2 n} .
\end{gathered}
$$


It is known that the problem (5.28) - (5.29) is solvable if and only if $S_{n} \geq 0$. A solution $\sigma$ of the problem (5.28) - (5.29) is called "exact" if

$$
\int t^{2 n} d \sigma(t)=s_{2 n}
$$

Singular truncated Hamburger moment problem has been studied in [16], [1], [1].

Theorem 5.5. ([16], [11]) Let $S_{n}=\left(s_{i+j}\right)_{i, j=0}^{n}-$ be a nonnegative block Hankel matrix $\left(s_{j} \in \mathbb{C}^{d \times d}\right)$. The following assertions are equivalent:

1) The problem (5.28) - (5.29) has an "exact" solution;

2) $\operatorname{ran}\left[\begin{array}{c}s_{n+1} \\ \vdots \\ s_{2 n}\end{array}\right] \subseteq \operatorname{ran} S_{n-1}$;

3) $S_{n}$ admits a nonnegative block Hankel extension $\widetilde{S}_{n}$.

The equivalence $(1) \Leftrightarrow(2)$ and $(2) \Leftrightarrow(3)$ were proved in [16] and [11], respectively.

Moreover, it was shown in 11 that if the conditions 1)-3) in Theorem 5.5 fail to hold then one can replace the right lower block $s_{2 n}$ in the matrix $S_{n}$ by $s_{2 n}^{\prime}$ in such a way that the new matrix $S_{n}{ }^{\prime}=\left(s_{i+j}{ }^{\prime}\right)_{i, j=0}^{n}$ with $s_{i}{ }^{\prime}=s_{i}$ for $i \leq 2 n-1$ satisfies 1$\left.)-3\right)$ in Theorem 5.5 and the sets $\mathcal{Z}\left(S_{n}\right)$ and $\mathcal{Z}\left(S_{n}^{\prime}\right)$ coincide.

In what follows it is supposed that $S_{n}$ satisfies the assumptions 1)-3) of Theorem 5.5. We will need also the following statement from [11.

Lemma 5.6. Let a block Hankel matrix $S_{n}=\left(s_{i+j}\right)_{i, j=0}^{n}$ satisfy the assumptions of Theorem 5.5 and let the matrix $T \in \mathbb{C}^{N \times N}(N=(n+1) d)$ be given by

$$
T=\left[\begin{array}{cccc}
0_{d} & I_{d} & & \\
& \ddots & \ddots & \\
& & \ddots & I_{d} \\
& & & 0_{d}
\end{array}\right] .
$$

Then there exists a matrix $X=X^{*} \in \mathbb{C}^{N \times N}$ of $\operatorname{rank} X=\operatorname{rank} S_{n}$ such that

$$
X S_{n} X=X, S_{n} X S_{n}=S_{n}, T \operatorname{Tan} X \subseteq \operatorname{ran} X .
$$

Let $\mathcal{X}$ be the space of vector polynomials $h(X)$ of the form (5.11) of formal degree $n$ and let the form $K(\cdot, \cdot)$ be given by (5.12). Define the operators $B_{1}, B_{2}: \mathcal{X} \rightarrow \mathcal{X}$ and $C_{1}, C_{2}: \mathcal{X} \rightarrow \mathcal{L}$ by (5.14). Then the data set $\left(B_{1}, B_{2}, C_{1}, C_{2}, K\right)$ satisfies the assumption (A1). Choosing the basis $1, X, \ldots, X^{n}$ in $\mathcal{X}$ one can identify $\mathcal{X}$ with $\mathbb{C}^{N}$ and then the form $K(\cdot, \cdot)$ is given by

$$
K(h, g)=\left(S_{n} h, g\right), \quad h, g \in \mathbb{C}^{N}, \quad N=(n+1) d .
$$

The operators $B_{1}, B_{2}$ and $C_{1}, C_{2}$ can be identified with their matrix representations in this basis

$$
\begin{gathered}
B_{1}=T, \quad B_{2}=I_{N} \\
C_{1}=\left[\begin{array}{llll}
0 & s_{0} & \ldots & s_{n-1}
\end{array}\right], \quad C_{2}=\left[\begin{array}{llll}
-I_{d} & 0 & \ldots & 0
\end{array}\right] .
\end{gathered}
$$


Then $B_{2}-\lambda B_{1}=I_{N}-\lambda T$ is invertible for all $\lambda \in \mathbb{C} \backslash\{0\}$

$$
G(\lambda)=\left[\begin{array}{l}
C_{1} \\
C_{2}
\end{array}\right]\left(I_{N}-\lambda T\right)^{-1}=\left[\begin{array}{l}
C_{1} \\
C_{2}
\end{array}\right]\left[\begin{array}{ccc}
I_{d} & \ldots & \lambda^{n} I_{d} \\
& \ddots & \vdots \\
& & I_{d}
\end{array}\right]
$$

Since the operators $B_{1}=T, B_{2}=I_{N}$ satisfy the assumption (U) and $\operatorname{ran} C_{2}=$ $\mathcal{L}$ the mapping $F: \mathcal{X} \rightarrow \mathcal{H}(\varphi, \psi)$ corresponding to the solution $\{\varphi, \psi\}$ of the $\operatorname{AIP}\left(B_{1}, B_{2}, C_{1}, C_{2}, K\right)$ is uniquely defined and any solution $\{\varphi, \psi\}$ of the AIP is equivalent to a pair $\left\{I_{d}, m(\lambda)\right\}$, where $m \in N^{d \times d}$. The corresponding AIP can be formulated as follows:

Find a Nevanlinna mvf $m \in N^{d \times d}$ such that:

(C1) $F h=\left[I_{d}-m(\lambda)\right] G(\lambda) h \in \mathcal{H}(m)$ for all $h \in \mathcal{X}$;

(C2) $\|F h\|_{\mathcal{H}(m)}^{2} \leq\left(S_{n} h, h\right)$ for all $h \in \mathcal{X}$.

Proposition 5.7. Let $m$ be a solution of the $A I P\left(B_{1}, B_{2}, C_{1}, C_{2}, S_{n}\right)$. Then $m$ admits the integral representation

$$
m(\lambda)=\int_{-\infty}^{\infty} \frac{d \sigma(t)}{t-\lambda}
$$

where $\sigma \in \mathcal{Z}\left(S_{n}\right)$. Conversely, if $\sigma \in \mathcal{Z}\left(S_{n}\right)$, then $m$ is a solution of the AIP.

Proof. Necessity. The same arguments as in the proof of Theorem 5.3 show that (C1) implies

$$
m(\lambda)+\frac{s_{0}}{\lambda}+\cdots+\frac{s_{n-1}}{\lambda^{n}}=O\left(\frac{1}{\lambda^{n+1}}\right) \quad(\lambda \widehat{\rightarrow} \infty) .
$$

Let $m(\lambda)$ have the integral representation (5.35) and let us set

$$
s_{j}^{(m)}=\int_{\mathbb{R}} t^{j} d \sigma(t) \quad(j=0,1, \ldots, 2 n) .
$$

It follows from (5.36) that

$$
s_{j}^{(m)}=s_{j} \text { for } j=0,1, \ldots, n-1 .
$$

The rest of the equalities (5.28) and the inequality (5.29) are implied by the condition (C2).

Let us show that for every polynomial $h(X)=\sum_{j=0}^{n} u_{j} X^{j}, \quad u_{j} \in \mathbb{C}^{d}$, the following equality holds

$$
\|F h\|_{\mathcal{H}(m)}^{2}=\sum_{j, k=0}^{n} u_{k}^{*} s_{j+k}^{(m)} u_{j} .
$$

Indeed, it follows from (5.25) that

$$
\begin{aligned}
(F h)(\lambda) & =\left[\begin{array}{llll}
m(\lambda) & \lambda m(\lambda)+s_{0} & \ldots & \lambda^{n} m(\lambda)+\lambda^{n-1} s_{0}+\cdots+s_{n-1}
\end{array}\right] u \\
& =\left[\begin{array}{llll}
\int_{\mathbb{R}} \frac{d \sigma(t)}{t-\lambda} & \int_{\mathbb{R}} \frac{t d \sigma(t)}{t-\lambda} & \cdots & \int_{\mathbb{R}} \frac{t^{n} d \sigma(t)}{t-\lambda}
\end{array}\right] u \in \mathcal{H}(m),
\end{aligned}
$$

where $u=\operatorname{col}\left(u_{0}, u_{1}, \ldots, u_{n}\right) \in \mathbb{C}^{(n+1) d}$. Due to [4, Theorem 2.5]

$$
\|F h\|_{\mathcal{H}(m)}^{2}=u^{*} S_{n}^{(m)} u,
$$


where

$$
S_{n}^{(m)}=\left[s_{i+j}^{(m)}\right]_{i, j=0}^{n} .
$$

Then the inequality $S_{n}^{(m)} \leq S_{n}$ in (C2) and (5.38) imply that (5.28) and (5.29) hold.

Sufficiency. Let $\sigma \in \mathcal{Z}\left(S_{n}\right)$ and let $m$ be defined by (5.35). Then it follows from (5.40) that (C1) holds.

The condition (C2) is implied by (5.41), (5.28) and (5.29), since

$$
\|F h\|_{\mathcal{H}(m)}^{2}=u^{*} S_{n}^{(m)} u \leq u^{*} S_{n} u, \quad u \in \mathbb{C}^{N} .
$$

In the regular case (when $\operatorname{det} S_{n} \neq 0$ ) the solution matrix $\Theta(\lambda)$ can be calculated by (4.38). Since $C_{1}^{+}=S_{n}^{-1} C_{1}^{*}$ and $C_{2}^{+}=S_{n}^{-1} C_{2}^{*}$ one obtains from (4.38) that

$$
\Theta(\lambda)=I_{2 d}-\lambda\left[\begin{array}{l}
C_{1} \\
C_{2}
\end{array}\right]\left(I_{\mathcal{H}}-\lambda T\right)^{-1} S_{n}^{-1}\left[\begin{array}{ll}
C_{2}^{*} & -C_{1}^{*}
\end{array}\right] .
$$

Then by Corollary 4.12 the formula (5.27) establishes the 1-1 correspondence between the set of all solutions $\sigma \in \mathcal{Z}\left(S_{n}\right)$ of the truncated moment problem (5.28)(5.29) and the set of all equivalence classes of Nevanlinna pairs $\{p, q\} \in \widetilde{N}^{d \times d}$.

In the singular case $\left(\operatorname{det} S_{n}=0\right.$ ) let us consider the matrix $X \in \mathbb{C}^{N \times N}$ which satisfies (5.31). Then the decomposition

$$
\mathcal{X}=\operatorname{ran} X \dot{+} \operatorname{ker} S_{n}
$$

satisfies the assumptions (A2), (A3), since $\operatorname{Tran} X \subseteq \operatorname{ran} X$, and the solution matrix $\Theta(\lambda)$ takes the form (4.38). Now, let us calculate the operators $C_{1}^{+}, C_{2}^{+}: \mathcal{L} \rightarrow \mathcal{H}$. For arbitrary $h=X g \in \operatorname{ran} X, u \in \mathcal{L}$ and $j=1,2$ one obtains

$$
\left(C_{j} X g, u\right)_{\mathbb{C}^{d}}=\left(X g, C_{j}^{*} u\right)_{\mathbb{C}^{N}}=\left(X g, S_{n} X C_{j}^{*} u\right)_{\mathbb{C}^{N}}=\left(X g, X C_{j}^{*} u\right)_{\mathcal{H}}
$$

Therefore, $C_{1}^{+}=X C_{1}^{*}, C_{2}^{+}=X C_{2}^{*}$, and the mvf $\Theta(\lambda)$ takes the form

$$
\Theta(\lambda)=\left(I_{\mathcal{L} \oplus \mathcal{L}}-\lambda\left[\begin{array}{l}
C_{1} \\
C_{2}
\end{array}\right]\left(I_{\mathcal{H}}-\lambda T\right)^{-1} X\left[\begin{array}{ll}
C_{2}^{*} & -C_{1}^{*}
\end{array}\right]\right) V
$$

where $V \in \mathbb{C}^{2 d \times 2 d}$ is a unitary matrix such that

$$
V\left(\{0\} \times \mathbb{C}^{\nu}\right)=\left[\begin{array}{c}
C_{1} \\
-C_{2}
\end{array}\right] \operatorname{ker} S_{n}=\left[\begin{array}{cccc}
0 & s_{1} & \ldots & s_{n-1} \\
I_{d} & 0 & \ldots & 0
\end{array}\right] \operatorname{ker} S_{n}
$$

and

$$
\nu=\operatorname{dim}\left[\begin{array}{cccc}
0 & s_{1} & \ldots & s_{n-1} \\
-I_{d} & 0 & \ldots & 0
\end{array}\right] \operatorname{ker} S_{n}
$$

Then by Corollary 4.12 the formula (5.27) establishes the 1-1 correspondence between the set of all solutions $\sigma \in \mathcal{Z}\left(S_{n}\right)$ of the truncated moment problem (5.28)(5.29) and the set of all equivalence classes of Nevanlinna pairs $\{p, q\} \in \widetilde{N}^{d \times d}$ of the form (4.35). 


\section{REFERENCES}

[1] V.M. Adamjan, I.M. Tkachenko, Solutions of the truncated matrix Hamburger moment problem according to M.G. Kreı̆n, Oper. Theory: Adv. Appl., 118 (2000), Birkhäuser Verlag, Basel, 35-51.

[2] D. Alpay, A. Dijksma, J. Rovnyak, and H.S.V. de Snoo, Schur functions, operator colligations, and reproducing kernel Pontryagin spaces, Oper. Theory: Adv. Appl., 96, Birkhäuser Verlag, Basel, 1997.

[3] D. Alpay, J. Ball, I. Gohberg, and L. Rodman, The two-sided residue interpolation in the Stieltjes class for matrix functions, Linear Algebra and its Appl., 208/209, (1994), 485-521.

[4] D. Alpay, I. Gohberg, Pairs of selfadjoint operators and their invariants, St. Petersburg Math. J., Vol 16 (2005), no. 1, 59-104.

[5] D. Alpay, H. Dym, Hilbert spaces of analytic functions, invers scattering and operator models, I. Integr, Equations and Operator Theory, 7 (1984), 589-641.

[6] D. Alpay, P. Bruinsma, A. Dijksma, and H.S.V. de Snoo, Interpolation problems, extensions of symmetric operators and reproducing kernel spaces. I, Oper. Theory: Adv. Appl., 50, Basel: Birkhäuser Verlag, 1991, 35-82.

[7] D.Z. Arov and L.Z. Grossman, Scattering matrices in the theory of unitary extensions of isometric operators, Math. Nachr., 157 (1992), 105-123.

[8] J. Ball, I. Gohberg, and L. Rodman, Interpolation of rational matrix functions, OT45, Birkhäuser Verlag, 1990.

[9] J.A. Ball, H.W. Helton, A Beurling-Lax theorem for the Lie group $U(m, n)$ which contains most classical interpolation theory, J. Operator Theory, 9 (1983), 107-142.

[10] Ch. Berg, Y. Chen, M.E.H. Ismail, Small eigenvalues of large Hankel matrices: The indeterminate case, Math. Scand. 91 (2002), no. 1, 67-81. MR1917682 (2003f:47042)

[11] V. Bolotnikov, On degenerate Hamburger moment problem and extensions of nonnegative Hankel block matrices, Integral Equations and Operator Theory, 25 (1996) no.3, 253-276.

[12] V. Bolotnikov, H. Dym, On degenerate interpolation, entropy and extremal problems for matrix Schur functions. Integral Equations Operator Theory 32 (1998), no. 4, 367-435.

[13] L.de Branges, Perturbation theory, J. Math. Anal.Appl., 57 (1977), 393-415.

[14] Bruinsma P., Degenerate interpolation problems for Nevanlinna pairs, Indag Math. N.S., $2(2), 1991,179-200$.

[15] V.M. Bruk. On a class of boundary value problems with spectral parameters in the boundary condition, Matem.Sb., 100 (1976), 210 - 216.

[16] R.E. Curto, L.A. Fialkow, Recursiveness, positivity, and truncated moment problems, Houston J. Math., 17 (1991), 603-635.

[17] V.A. Derkach,M.M. Malamud, The extension theory of hermitian operators and the moment problem, J.of Math.Sci. 73 (1995) No.2, 141-242.

[18] V.A. Derkach, S. Hassi, M.M. Malamud, H.S.V. de Snoo, Generalized resolvents of symmetric operators and admissibility, Methods of Functional Analysis and Topology, 6 (2000), 24-55.

[19] V.A. Derkach, S. Hassi, M.M. Malamud, H.S.V. de Snoo, Boundary relations and their Weyl families, Trans.Amer.Math.Soc. 358 (2006) 5351-5400.

[20] H. Dym, J-contractive matrix functions, reproducing kernel Hilbert spaces and interpolation, CBMS Regional Series in Math., vol.71, Providence, RI, 1989.

[21] H. Dym, Riccati equations and bitangential interpolation problems with singular Pick matrices. Fast algorithms for structured matrices: theory and applications (South Hadley, MA, 2001), 361-391, Contemp. Math., 323, Amer. Math. Soc., Providence, RI, 2003.

[22] V. I. Gorbachuk and M. L. Gorbachuk, Boundary value problems for operator differential equations. Translated and revised from the 1984 Russian original. Mathematics and its Applications (Soviet Series), 48. Kluwer Academic Publishers Group, Dordrecht, 1991. xii +347 pp. 
[23] I.P. Fedchina, Criteria for the solvability of Nevanlinna-Pick tangent problem, Matem. Issl. 7 (1972), no. 4 (26), 213-227.

[24] I.P. Fedchina, Description of solutions of Nevanlinna-Pick tangent problem, Dokl. Akad. Nauk Armjan.SSR, ser. mat., 60 (1975), 37-42.

[25] V.E. Katsnelson, A.Ya. Kheifets and P.M. Yuditskii, The abstract interpolation problem and extension theory of isometric operators, in: "Operators in Spaces of Functions and Problems in Function Theory", Kiev, Naukova Dumka, 1987, 83-96 (Russian).

[26] A.Ya. Kheifets and P.M. Yuditskii, An analysis and extension of V.P. Potapov's approach to interpolation problems with applications to the generalized bi-tangential Schur-Nevanlinna-Pick problem and $J$-inner-outer factorization, Operator Theory: Adv.Appl., 72, Birkhauser, Basel, 1994. - P.133-161.

[27] I.V. Kovalishina and V.P. Potapov, Indefinite metric in Nevanlinna-Pick problem, Dokl. Akad. Nauk Armjan.SSR, ser. mat., 59 (1974) 17-22.

[28] A. Kheifets, Generalized bitangential Schur-Nevanlinna-Pick problem and related with it Parseval equality, Teor. funkcij i pril., Kharkov, 54 (1990), 89-96.

[29] A. Kheifets, Hamburger Moment problem: Parseval equality and A-singularity, J. Funct. Analysis, 141, (1996) 374-420.

[30] M.G. Kreĭn, On Hermitian operators with defect indices equal to one, Dokl. Akad. Nauk SSSR, 43, No.8 (1944), 339-342.

[31] M.G. Kreı̆n, Fundamental aspects of the representation theory of Hermitian operators with deficiency index $(m, m)$, Ukrain. Math. Zh., 1 (1949), 3-66 (Russian); (English translation: Amer. Math. Soc. Transl., (2) 97 (1970), 75-143).

[32] M.G. Kreı̆n and H.Langer, Über die verallgemeinerten Resolventen und die characteristische Function eines isometrischen Operators im Raume $\Pi_{\kappa}$, Hilbert space Operators and Operator Algebras (Proc.Intern.Conf.,Tihany, 1970 ); Colloq.Math.Soc.Janos Bolyai, vol.5, North-Holland, Amsterdam, 353-399, 1972.

[33] M.G. Krein and Sh.N. Saakyan, Some new results in the theory of resolvent matrices of Hermitian operators, Dokl. Akad. Nauk SSSR, 169, No.1 (1966) 657-660.

[34] S. Kupin, Lifting theorem as a special case of abstract interpolation problem, J. Anal. Appl., 15 (1996), no. 4, 789-798.

[35] M.M. Malamud, On the formula of generalized resolvents of a nondensely defined Hermitian operator, Ukr. Mat. Zh., 44 No. 2, (1992), 1658-1688.

[36] A.A. Nudel'man, On a new problem of moment type, Dokl.Akad.Nauk SSSR 233 (1977), 792-795; Soviet Math. Dokl., 18 (1977), 507-510.

[37] V.P. Potapov, Multiplicative structure of $J$-nonexpanding matrix functions, Trudy Mosk.Matem. Obsch., 4, (1955) 125-236.

[38] A.V. Štraus, Extensions and generalized resolvents of a symmetric operator which is not densely defined, Izv. Akad. Nauk. SSSR, Ser. Mat., 34 (1970), 175-202 (Russian) [English translation: Math. USSR-Izvestija, 4 (1970), 179-208].

Department of Mathematics, Donetsk National University, Universitetskaya Str. 24, 83055 Donetsk, Ukraine

E-mail address: derkach.v@gmail.com 